\title{
A Matrix Isolation Spectroscopic and Quantum Chemical Study of Fumaric and Maleic Acid
}

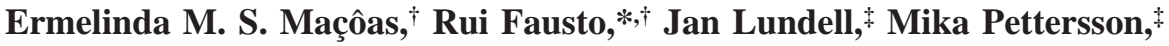 \\ Leonid Khriachtchev, ${ }^{*}$ and Markku Räsänen* \\ Department of Chemistry - CQC, University of Coimbra, P-3049 Coimbra, Portugal, and Laboratory of \\ Physical Chemistry, University of Helsinki, P.O. Box 55 (A.I. Virtasen aukio 1), FIN-00014 Helsinki, Finland
}

Received: October 16, 2000; In Final Form: January 29, 2001

\begin{abstract}
Relative energies and vibrational spectra of buthenedioic acid $[(E)-$ and $(Z)-\mathrm{HOOC}-\mathrm{CH}=\mathrm{CH}-\mathrm{COOH}$; fumaric and maleic acids] were calculated using Hartree-Fock (HF), Møller-Plesset to 2nd order (MP2), and B3LYP Density Functional Theory (DFT) levels of approximation. Computationally the lowest energy conformer of maleic acid has a nearly planar structure with an $\mathrm{OH} \cdots \mathrm{O}=\mathrm{C}$ intramolecular hydrogen bond closing a seven member $\mathrm{O}=\mathrm{C}-\mathrm{C}(\mathrm{H})=\mathrm{C}(\mathrm{H})-\mathrm{C}-\mathrm{O}-\mathrm{H}$ ring. Second conformer of maleic acid adopts a cis arrangement around the $\mathrm{C}-\mathrm{O}$ bonds and exhibits the two carbonyl bonds in a nearly orthogonal arrangement with one carboxylic group in the plane containing the carbon atoms. Fumaric acid has three planar conformers with relative energies within $5 \mathrm{~kJ} \mathrm{~mol}^{-1}$. These conformers exhibit a cis arrangement around the $\mathrm{C}-\mathrm{O}$ bond and differ by the relative conformation of their $\mathrm{O}=\mathrm{C}-\mathrm{C}=\mathrm{C}$ axes. The infrared spectra of the studied compounds isolated in a solid argon matrix feature the most stable conformers. Narrowband tunable irradiation in the near-infrared region $\left(6901 \mathrm{~cm}^{-1}\right)$ was found to promote a conversion of the second most stable conformer of maleic acid into the most stable conformer.
\end{abstract}

\section{Introduction}

Both $(E)$ - and (Z)-buthenedioic acids (fumaric and maleic acids) have important practical applications in polymer industry ${ }^{1-4}$ and in medicine, in particular as anti-fungicides and antitumoral agents. ${ }^{5-9}$ In a more fundamental perspective, studies on their structural and spectroscopic properties is important, since they are the simplest dicarboxylic acids exhibiting a carbon-carbon double bond. Despite the wide range of application of these carboxylic acids, to the best of our knowledge, there are no studies on monomeric fumaric and maleic acids available, the existing information concerning essentially with the structure of their crystalline phases. ${ }^{10-12}$

Detailed structural and vibrational studies were reported for the monocarboxylic parent molecule, propenoic acid (acrylic acid, $\left.\mathrm{CH}_{2}=\mathrm{CHCOOH}\right),{ }^{13}$ and the related molecules, $(Z)$ - and (E)-3-methyl-propenoic [(Z)- and (E)-crotonic acid, $\left.\mathrm{CH}_{3} \mathrm{CH}=\mathrm{CHCOOH}\right] .{ }^{14,15}$ These studies revealed that these molecules exist both in the gas phase and in solid argon in two conformational states differing by internal rotation around the $\mathrm{C}_{\alpha}-\mathrm{C}$ bond and having similar energies $\left[\Delta E_{(\mathrm{s}-\text { trans })-(\mathrm{s}-\mathrm{cis})} \approx 3\right.$ $\left.\mathrm{kJ} \mathrm{mol}^{-1}\right]$. The s-cis conformer $\left(\mathrm{C}=\mathrm{C}_{\alpha}-\mathrm{C}=\mathrm{O}\right.$ dihedral equals to $0^{\circ}$ ) was found to be the conformational ground state for the three molecules. ${ }^{13-15}$ Ultraviolet irradiation $\left(\lambda_{\text {exc }}=243 \mathrm{~nm}\right)$ of matrix isolated $(E)$-crotonic acid was found to promote conformational isomerization from the s-cis conformer to the s-trans conformer $\left(\mathrm{C}=\mathrm{C}_{\alpha}-\mathrm{C}=\mathrm{O}\right.$ dihedral equals to $\left.180^{\circ}\right)$ as well as conversion to $(Z)$-crotonic acid, the latter process being noticeably more efficient than the first one. ${ }^{14,15}$

In this article, we present a detailed study of the conformers of monomeric maleic and fumaric acids combining ab initio

* To whom all correspondence should be addressed.

$\dagger$ Department of Chemistry.

$\doteqdot$ Laboratory of Physical Chemistry. and Density Functional Theory (DFT) calculations and matrix isolation vibrational spectroscopy with selective near-infrared induced rotamerization. In particular, the main factors which determine the stability of different conformers, their vibrational signature and the photoinduced interconversion processes between the lowest energy species are analyzed.

\section{Experimental and Computational Details}

The matrix samples were prepared by continuously flushing a copper tube containing maleic or fumaric acid (Aldrich, $99+\%$ ), heated to 353 and $408 \mathrm{~K}$, respectively, with high purity argon (Aga, 99.9999\%). The gaseous mixtures were deposited onto a cooled CsI window in a close-cycle helium cryostat (APD, DE202A) at 7.5 K. In our samples, monomeric species were almost exclusively formed, which was achieved by choosing an appropriate temperature of the substance container and optimizing the matrix gas flow rate. The IR spectra (4000$400 \mathrm{~cm}^{-1}$ ) were measured with a Nicolet SX-60 FTIR spectrometer equipped with a liquid nitrogen cooled MCT detector and a $\mathrm{KBr}$ beam splitter, with spectral resolutions of 0.25 or $1.0 \mathrm{~cm}^{-1}$. Typically 200 interferograms were coadded.

Photoexcitation was performed using a tunable pulsed nearinfrared (NIR) radiation provided by an optical parametric oscillator (OPO Sunlite, Continuum) pumped by an Nd:YAG solid-state laser (Powerlite 9010, Continuum). The pulse duration of the NIR irradiation was ca.5 ns with a line width of $\sim 0.1 \mathrm{~cm}^{-1}$. The NIR radiation wavenumber was established with an accuracy better than $1 \mathrm{~cm}^{-1}$ by a Burleigh WA-4500 wavemeter used to control the OPO signal radiation wavelength. Broadband IR irradiation and UV excitation of fumaric acid were under-taken using the globar of the spectrometer and the $\mathrm{Nd}$ :YAG laser $(\lambda=266 \mathrm{~nm})$, respectively.

The ab initio and DFT calculations ${ }^{16-18}$ were performed with 


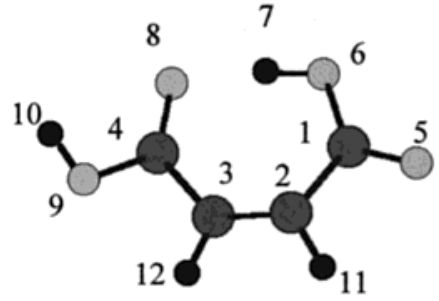

I

$$
1.5 \mathrm{HF}
$$

$0.0 \mathrm{MP} 2$

0.0 B3LYP

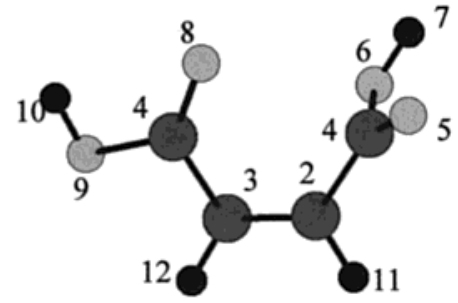

II $\quad 0.0$

1.3

14.6

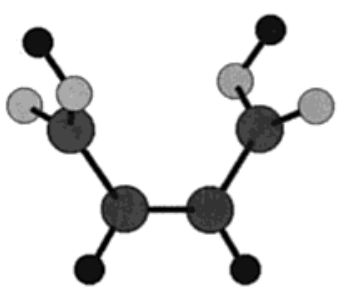

III $\max$.

5.8

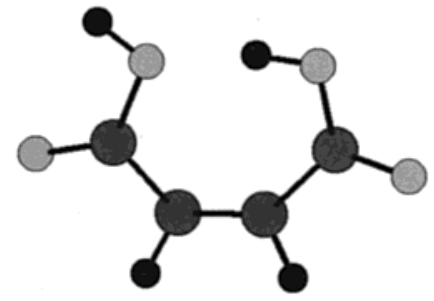

IV
22.7

20.3

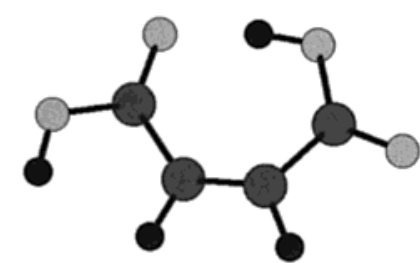

V $\quad 32.0$

27.5

24.7

Figure 1. Structure and relative energies $\left(\mathrm{kJ} \mathrm{mol}^{-1}\right)$ of most stable conformers of maleic acid predicted at three different theoretical levels. The relative energies account for the zero-point-vibrational-energy correction.

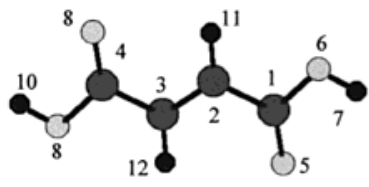

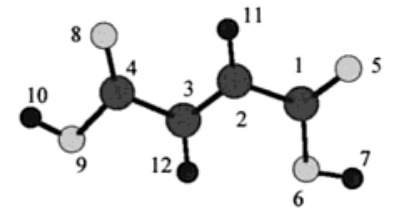

II $\quad 2.0$

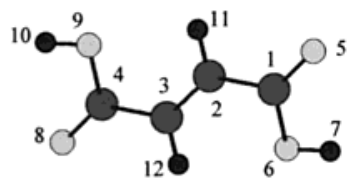

III $\quad 3.5$

0.0 B 3 LYP

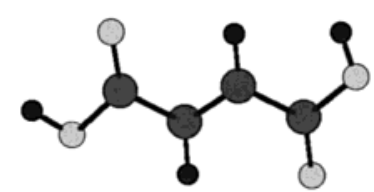

IV $\quad 29.5$

26.7

24.3

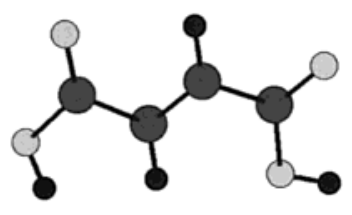

V $\quad 33.1$

30.3

28.1

Figure 2. Structure and relative energies $\left(\mathrm{kJ} \mathrm{mol}^{-1}\right)$ of the most stable conformers of fumaric acid predicted at three different theoretical levels. The relative energies account for the zero-point-vibrational-energy correction.

GAUSSIAN 92/DFT or $94^{19}$ on a DEC ALPHA 7000 computer at the Center of Informatics of the University of Coimbra and on a SGI Origin 2000 at the CSC-Center for Scientific Computing, Ltd. (Espoo, Finland). Geometry optimizations were performed at three different levels of theory [Hartree-Fock (HF), Møller-Plesset to 2nd order (MP2), and B3LYP Density Functional Theory (DFT)] using the 6-31G(d,p) basis set. ${ }^{20,21}$ Vibrational calculations were performed to ensure that the found stationary points were true minima and not transition states. We report here only the B3LYP/6-31G(d,p) frequencies in consonance with our previous work on malonic acid. ${ }^{22}$ The force constants (symmetry internal coordinates) to be used in the normal coordinate analysis were obtained from the DFT
Cartesian harmonic force constants using the program TRANSFORMER. ${ }^{23}$ This program was also used to prepare the input data for the normal coordinate analysis programs used in this study (BUILD-G, and VIBRAT). ${ }^{24}$

\section{Results and Discussion}

Molecular Geometries and Energetics. To find the most stable conformations of maleic and fumaric acids we performed a systematic search on their $\mathrm{HF} / 6-31 \mathrm{G}(\mathrm{d})$ potential energy surfaces (PES). These calculations are relatively inexpensive in computational terms and yield reasonable structural data for similar compounds. ${ }^{25}$ The HF/6-31G(d) calculations were then 
TABLE 1: MP2 and B3LYP Geometrical Parameters of Maleic Acid Conformers Obtained Using the 6-31G(d,p) Basis Set

\begin{tabular}{|c|c|c|c|c|}
\hline \multirow{3}{*}{$\begin{array}{l}\text { geometrical } \\
\text { parameters }\end{array}$} & \multicolumn{4}{|c|}{ conformer } \\
\hline & \multicolumn{2}{|c|}{ MP2 } & \multicolumn{2}{|c|}{ B3LYP } \\
\hline & I & II & I & II \\
\hline bond (pm) & & & & \\
\hline $\mathrm{C}_{1}=\mathrm{O}_{5}$ & 122.2 & 121.7 & 121.5 & 121.0 \\
\hline $\mathrm{C}_{1}-\mathrm{O}_{6}$ & 133.7 & 135.2 & 133.0 & 134.6 \\
\hline $\mathrm{O}_{6}-\mathrm{H}_{7}^{a}$ & 98.3 & 97.2 & 99.0 & 97.3 \\
\hline $\mathrm{C}_{2}-\mathrm{C}_{1}$ & 150.5 & 149.6 & 151.1 & 150.0 \\
\hline $\mathrm{C}_{2}-\mathrm{H}_{11}$ & 108.2 & 108.3 & 108.6 & 108.7 \\
\hline $\mathrm{C}_{2}=\mathrm{C}_{3}$ & 135.0 & 134.1 & 134.6 & 133.7 \\
\hline $\mathrm{C}_{3}-\mathrm{H}_{12}$ & 108.2 & 108.1 & 108.6 & 108.5 \\
\hline $\mathrm{C}_{3}-\mathrm{C}_{4}$ & 147.6 & 148.0 & 147.6 & 148.3 \\
\hline $\mathrm{C}_{4}=\mathrm{O}_{8}{ }^{a}$ & 123.1 & 122.1 & 122.6 & 121.4 \\
\hline $\mathrm{C}_{4}-\mathrm{O}_{9}$ & 134.6 & 135.7 & 134.3 & 135.5 \\
\hline $\mathrm{O}_{9}-\mathrm{H}_{10}$ & 97.2 & 97.1 & 97.3 & 97.2 \\
\hline $\begin{array}{l}\qquad \mathrm{C}_{4}=\mathrm{O}_{8} \cdots \mathrm{H}_{7}-\mathrm{O}_{6} \\
\text { angle (deg) }\end{array}$ & 168.0 & & 164.7 & \\
\hline $\mathrm{C}_{2}-\mathrm{C}_{1}=\mathrm{O}_{5}$ & 117.6 & 123.7 & 117.4 & 122.8 \\
\hline $\mathrm{O}_{5}=\mathrm{C}_{1}-\mathrm{O}_{6}$ & 121.8 & 124.4 & 122.1 & 124.2 \\
\hline $\mathrm{C}_{1}-\mathrm{O}_{6}-\mathrm{H}_{7}$ & 111.4 & 105.6 & 112.0 & 106.3 \\
\hline $\mathrm{C}_{1}-\mathrm{C}_{2}=\mathrm{C}_{3}$ & 133.9 & 124.5 & 133.8 & 126.2 \\
\hline $\mathrm{C}_{1}-\mathrm{C}_{2}-\mathrm{H}_{11}$ & 109.9 & 115.5 & 109.5 & 114.2 \\
\hline $\mathrm{C}_{4}-\mathrm{C}_{3}=\mathrm{C}_{2}$ & 128.2 & 121.6 & 127.9 & 122.8 \\
\hline $\mathrm{C}_{4}-\mathrm{C}_{3}-\mathrm{H}_{12}$ & 114.0 & 117.5 & 113.8 & 116.7 \\
\hline $\mathrm{C}_{3}-\mathrm{C}_{4}=\mathrm{O}_{8}$ & 127.6 & 125.7 & 127.3 & 126.0 \\
\hline $\mathrm{O}_{8}=\mathrm{C}_{4}-\mathrm{O}_{9}$ & 121.8 & 123.4 & 121.3 & 122.9 \\
\hline $\begin{array}{c}\mathrm{C}_{4}-\mathrm{O}_{9}-\mathrm{H}_{10} \\
\text { dihedral angle (deg) }\end{array}$ & 106.4 & 105.7 & 107.0 & 106.2 \\
\hline $\mathrm{O}_{5}=\mathrm{C}_{1}-\mathrm{O}_{6}-\mathrm{H}_{7}$ & 180.0 & -5.5 & 180.0 & -5.5 \\
\hline $\mathrm{O}_{5}=\mathrm{C}_{1}-\mathrm{C}_{2}-\mathrm{C}_{3}$ & 180.0 & 105.9 & 180.0 & 109.8 \\
\hline $\mathrm{C}_{1}-\mathrm{C}_{2}-\mathrm{C}_{3}-\mathrm{C}_{4}$ & 0.0 & 1.1 & 0.0 & 1.2 \\
\hline $\mathrm{O}_{8}=\mathrm{C}_{4}-\mathrm{O}_{9}-\mathrm{H}_{10}$ & 0.0 & 0.1 & 0.0 & 0.1 \\
\hline $\mathrm{O}_{8}=\mathrm{C}_{4}-\mathrm{C}_{3}-\mathrm{C}_{2}$ & 0.0 & -8.6 & 0.0 & -7.4 \\
\hline $\mathrm{H}_{11}-\mathrm{C}_{2}-\mathrm{C}_{3}-\mathrm{H}_{12}$ & 0.0 & 1.6 & 0.0 & -1.8 \\
\hline
\end{tabular}

${ }^{a} \mathrm{C}_{4}=\mathrm{O}_{8}$ and $\mathrm{O}_{6}-\mathrm{H}_{7}$ bond lengths in conformer I clearly show the intermolecular $\mathrm{O}_{6}-\mathrm{H}_{7} \cdots \mathrm{O}_{4}=\mathrm{C}_{8}$ hydrogen bond in this conformer as they are clearly longer than in the free $\mathrm{O}-\mathrm{H}$ and $\mathrm{C}=\mathrm{O}$ bonds in conformer II

used as the first estimate to the MP2 and DFT calculations. In these preliminary calculations, the $\mathrm{O}=\mathrm{C}-\mathrm{C}=\mathrm{C}$ dihedral angles of the initial structures submitted to geometry optimization were varied by $60^{\circ}$ within the range $0-360^{\circ}$ and the initial $\mathrm{O}=\mathrm{C}-\mathrm{O}-\mathrm{H}$ dihedral angles were made equal to $0^{\circ}$ or $180^{\circ}$ (all nonequivalent-by-symmetry combinations of the dihedral angles were considered).

Maleic Acid. Following the indications provided by the preliminary $\mathrm{HF} / 6-31 \mathrm{G}(\mathrm{d})$ calculations, five conformers of maleic acid are found at the MP2 and B3LYP levels. These conformers are shown in Figure 1, where their relative energies are also given [complete structural results obtained at the $\mathrm{HF} / 6-31 \mathrm{G}(\mathrm{d})$ level of theory and MP2 and B3LYP geometries for the highest energy conformers can be received from the authors upon request]. Table 1 shows the MP2 and B3LYP calculated geometries for the two lowest energy conformers. The most stable conformer (I) is nearly planar, with an $\mathrm{OH} \cdots \mathrm{O}=\mathrm{C}$ intramolecular hydrogen bond closing the seven member $\mathrm{O}=\mathrm{C}-\mathrm{C}(\mathrm{H})=\mathrm{C}(\mathrm{H})-\mathrm{C}-\mathrm{O}-\mathrm{H}$ ring. The second most stable conformer (II) is nonplanar with cis arrangements around the $\mathrm{C}-\mathrm{O}$ bonds. The two carbonyl bonds of this conformer are nearly orthogonal with one carboxylic group in the plane containing the carbon atoms.

The energy difference between conformer II and the conformational ground state is estimated to be $14.6 \mathrm{~kJ} \mathrm{~mol}^{-1}$ at the B3LYP level, whereas the MP2 calculations give a considerably smaller difference $\left(1.3 \mathrm{~kJ} \mathrm{~mol}^{-1}\right)$. As shown later, the MP2 result
TABLE 2: MP2 and B3LYP Geometrical Parameters of Fumaric Acid Conformers Obtained Using the 6-31G(d,p) Basis Set

\begin{tabular}{|c|c|c|c|c|c|c|}
\hline \multirow{3}{*}{$\begin{array}{l}\text { geometrical } \\
\text { parameters }\end{array}$} & \multicolumn{6}{|c|}{ conformers } \\
\hline & \multicolumn{3}{|c|}{ MP2 } & \multicolumn{3}{|c|}{ B3LYP } \\
\hline & I & II & III & I & II & III \\
\hline \multicolumn{7}{|l|}{ bond (pm) } \\
\hline $\mathrm{C}_{1}=\mathrm{O}_{5}$ & 122.0 & 122.1 & 122.1 & 121.4 & 121.3 & 121.4 \\
\hline $\mathrm{C}_{1}-\mathrm{O}_{6}$ & 135.7 & 136.0 & 135.9 & 135.3 & 135.5 & 135.4 \\
\hline $\mathrm{O}_{6}-\mathrm{H}_{7}$ & 97.2 & 97.1 & 97.1 & 97.2 & 97.2 & 97.2 \\
\hline $\mathrm{C}_{2}-\mathrm{C}_{1}$ & 148.2 & 147.8 & 147.9 & 148.5 & 148.2 & 148.3 \\
\hline $\mathrm{C}_{2}-\mathrm{H}_{11}$ & 108.1 & 108.2 & 108.0 & 108.5 & 108.5 & 108.4 \\
\hline $\mathrm{C}_{2}-\mathrm{C}_{3}$ & 134.2 & 134.2 & 134.3 & 133.7 & 133.8 & 133.9 \\
\hline $\mathrm{C}_{3}-\mathrm{H}_{12}$ & 108.1 & 108.0 & 108.0 & 108.5 & 108.4 & 108.4 \\
\hline $\mathrm{C}_{3}-\mathrm{C}_{4}$ & 148.2 & 148.2 & 147.9 & 148.5 & 148.6 & 148.3 \\
\hline $\mathrm{C}_{4}=\mathrm{O}_{8}$ & 122.0 & 122.0 & 122.1 & 121.4 & 121.3 & 121.4 \\
\hline $\mathrm{C}_{4}-\mathrm{O}_{9}$ & 135.7 & 135.7 & 135.9 & 135.3 & 135.4 & 135.4 \\
\hline $\mathrm{O}_{9}-\mathrm{H}_{10}$ & 97.2 & 97.2 & 97.1 & 97.2 & 97.2 & 97.2 \\
\hline \multicolumn{7}{|l|}{ angle (deg) } \\
\hline $\mathrm{C}_{2}-\mathrm{C}_{1}=\mathrm{O}_{5}$ & 125.7 & 123.9 & 123.8 & 125.6 & 123.6 & 123.4 \\
\hline $\mathrm{O}_{5}=\mathrm{C}_{1}-\mathrm{O}_{6}$ & 123.5 & 123.2 & 123.2 & 123.2 & 123.0 & 123.0 \\
\hline $\mathrm{C}_{1}-\mathrm{O}_{6}-\mathrm{H}_{7}$ & 105.9 & 105.4 & 105.4 & 106.3 & 106.0 & 106.1 \\
\hline $\mathrm{C}_{1}-\mathrm{C}_{2}=\mathrm{C}_{3}$ & 120.1 & 123.7 & 123.3 & 120.6 & 124.3 & 124.0 \\
\hline $\mathrm{C}_{1}-\mathrm{C}_{2}-\mathrm{H}_{11}$ & 119.0 & 115.8 & 115.1 & 118.5 & 115.2 & 114.4 \\
\hline $\mathrm{C}_{4}-\mathrm{C}_{3}=\mathrm{C}_{2}$ & 120.1 & 119.6 & 123.3 & 120.6 & 120.2 & 124.0 \\
\hline $\mathrm{C}_{4}-\mathrm{C}_{3}-\mathrm{H}_{12}$ & 119.0 & 118.4 & 115.1 & 118.5 & 117.9 & 114.1 \\
\hline $\mathrm{C}_{3}-\mathrm{C}_{4}=\mathrm{O}_{8}$ & 125.7 & 125.8 & 123.8 & 125.6 & 125.7 & 123.4 \\
\hline $\mathrm{O}_{8}=\mathrm{C}_{4}-\mathrm{O}_{9}$ & 123.5 & 123.5 & 123.2 & 123.2 & 123.2 & 123.0 \\
\hline $\mathrm{C}_{4}-\mathrm{O}_{9}-\mathrm{H}_{10}$ & 105.9 & 105.8 & 105.4 & 106.3 & 106.3 & 106.1 \\
\hline \multicolumn{7}{|l|}{ dihedral angle (deg) } \\
\hline $\mathrm{O}_{5}=\mathrm{C}_{1}-\mathrm{O}_{6}-\mathrm{H}_{7}$ & 0.0 & 0.0 & 0.0 & 0.0 & 0.0 & 0.0 \\
\hline $\mathrm{O}_{5}=\mathrm{C}_{1}-\mathrm{C}_{2}-\mathrm{C}_{3}$ & 0.0 & 180.0 & 180.0 & 0.0 & 180.0 & 180.0 \\
\hline $\mathrm{C}_{1}-\mathrm{C}_{2}-\mathrm{C}_{3}-\mathrm{C}_{4}$ & 180.0 & 180.0 & 180.0 & 180.0 & 180.0 & 180.0 \\
\hline $\mathrm{O}_{8}=\mathrm{C}_{4}-\mathrm{O}_{9}-\mathrm{H}_{10}$ & 0.0 & 0.0 & 0.0 & 0.0 & 0.0 & 0.0 \\
\hline $\mathrm{O}_{8}=\mathrm{C}_{4}-\mathrm{C}_{3}-\mathrm{C}_{2}$ & 0.0 & 0.0 & 180.0 & 0.0 & 0.0 & 180.0 \\
\hline $\mathrm{H}_{11}-\mathrm{C}_{2}=\mathrm{C}_{3}-\mathrm{H}_{12}$ & 180.0 & 180.0 & 180.0 & 180.0 & 180.0 & 180.0 \\
\hline
\end{tabular}

agrees much better with the experimentally determined conformer energy difference $\left(1.7 \mathrm{~kJ} \mathrm{~mol}^{-1}\right)$. These computational results follow the trends previously observed for other dicarboxylic acids where intramolecular hydrogen bonding is important, e.g., 1,3-propanedioic acid. ${ }^{22}$ At least for this kind of molecules, the B3LYP calculations tend to overestimate the stabilization energy due to difficulties in describing the intramolecular hydrogen bonding properly. Noteworthy, the HF calculations failed to reproduce the correct energy order of conformers I and II, predicting conformer II as the most stable form $\left(\Delta E=-1.5 \mathrm{~kJ} \mathrm{~mol}^{-1}\right)$. This result is a direct consequence of the inappropriate description of the intramolecular hydrogen bond present in conformer I, and it reinforces our previous conclusions concerning the importance of using higher theoretical levels. ${ }^{22,25,26}$ The relative importance of the intramolecular hydrogen bond, as shown by the three theoretical approaches used here, is also clearly evidenced by the calculated values for the $\mathrm{OH} \cdots \mathrm{O}=\mathrm{C}$ distance, which decrease from $\mathrm{HF}$ (174.4 pm) to MP2 (168.0 pm) and B3LYP (164.7 pm).

Conformers III $-\mathrm{V}$ are predicted by the MP2 calculations to have considerably higher energies than the two most stable forms. This is mostly due to (i) more important repulsive interactions between the lone electron pairs of the carboxylic oxygen atoms and reduced $\pi$-delocalization (as in conformer III), (ii) repulsions between hydrogen atoms (as in conformer $\mathrm{V}$, where the $\mathrm{OH}_{10} \cdots \mathrm{H}_{12}$ repulsion is prevalent), and (iii) presence of trans arrangements around the $\mathrm{C}-\mathrm{O}$ bonds ${ }^{27}$ which are not energetically compensated by strong intramolecular hydrogen bonding (as in conformers IV and V).

Fumaric Acid. For fumaric acid, $\mathrm{OH} \cdots . \mathrm{O}=\mathrm{C}$ intramolecular hydrogen bonding involving the two carboxylic groups is not possible and the three computational approaches used (HF, MP2, 
TABLE 3: Definition of the Internal Symmetry Coordinates Used in the normal Coordinate Analysis of Maleic and Fumaric Acids

\begin{tabular}{|c|c|c|c|c|c|}
\hline \multirow[b]{2}{*}{ coordinate } & \multirow[b]{2}{*}{ symmetry $^{a}$} & \multicolumn{2}{|c|}{$\begin{array}{l}\text { approximate } \\
\text { description }^{d}\end{array}$} & \multicolumn{2}{|l|}{ definition $^{e}$} \\
\hline & & $C_{2 h}^{b}$ & $C_{s} / C_{1}{ }^{c}$ & $C_{2 h}^{b}$ & $C_{s} / C_{1}^{c}$ \\
\hline $\mathrm{S} 1$ & $B_{u}$ & $v \mathrm{C}=\mathrm{O}$ a. & $v \mathrm{C}=\mathrm{O}$ & $\left(v \mathrm{C}_{1}=\mathrm{O}_{5}\right)-\left(v \mathrm{C}_{4}=\mathrm{O}_{8}\right)$ & $v \mathrm{C}_{1}=\mathrm{O}_{5}$ \\
\hline $\mathrm{S} 2$ & $A_{g}$ & $v \mathrm{C}=\mathrm{O}$ & $v \mathrm{C}=\mathrm{O}^{\prime}$ & $\left(v \mathrm{C}_{1}=\mathrm{O}_{5}\right)+\left(v \mathrm{C}_{4}=\mathrm{O}_{8}\right)$ & $v \mathrm{C}_{4}=\mathrm{O}_{8}$ \\
\hline $\mathrm{S} 3$ & $B_{u}$ & $v \mathrm{C}-\mathrm{O}$ a & $v \mathrm{C}-\mathrm{O}$ & $\left(v \mathrm{C}_{1}-\mathrm{O}_{6}\right)-\left(v \mathrm{C}_{4}-\mathrm{O}_{9}\right)$ & $v \mathrm{C}_{1}-\mathrm{O}_{6}$ \\
\hline S4 & $A_{g}$ & $v \mathrm{C}-\mathrm{O}$ & $v \mathrm{C}-\mathrm{O}^{\prime}$ & $\left(v \mathrm{C}_{1}-\mathrm{O}_{6}\right)+\left(v \mathrm{C}_{4}-\mathrm{O}_{9}\right)$ & $v \mathrm{C}_{4}-\mathrm{O}_{9}$ \\
\hline S5 & $B_{u}$ & $v \mathrm{O}-\mathrm{H}$ a. & $v \mathrm{O}-\mathrm{H}$ & $\left(v \mathrm{O}_{6}-\mathrm{H}_{7}\right)-\left(v \mathrm{O}_{9}-\mathrm{H}_{10}\right)$ & $v \mathrm{O}_{6}-\mathrm{H}_{7}$ \\
\hline S6 & $A_{g}$ & $v \mathrm{O}-\mathrm{H}$ & $v \mathrm{O}-\mathrm{H}^{\prime}$ & $\left(v \mathrm{O}_{6}-\mathrm{H}_{7}\right)+\left(v \mathrm{O}_{9}-\mathrm{H}_{10}\right)$ & $v \mathrm{O}_{9}-\mathrm{H}_{10}$ \\
\hline S7 & $B_{u}^{\circ}$ & $v \mathrm{C}-\mathrm{C}$ a. & $v \mathrm{C}-\mathrm{C}$ & $\left(v \mathrm{C}_{1}-\mathrm{C}_{2}\right)-\left(v \mathrm{C}_{3}-\mathrm{C}_{4}\right)$ & $v \mathrm{C}_{1}-\mathrm{C}_{2}$ \\
\hline S8 & $A_{g}$ & $v \mathrm{C}-\mathrm{C}$ & $v \mathrm{C}-\mathrm{C}^{\prime}$ & $\left(v \mathrm{C}_{1}-\mathrm{C}_{2}\right)+\left(v \mathrm{C}_{3}-\mathrm{C}_{4}\right)$ & $v \mathrm{C}_{3}-\mathrm{C}_{4}$ \\
\hline S9 & $B_{u}$ & $v \mathrm{C}-\mathrm{H}$ a. & $v \mathrm{C}-\mathrm{H}$ & $\left(v \mathrm{C}_{2}-\mathrm{H}_{12}\right)-\left(v \mathrm{C}_{3}-\mathrm{H}_{11}\right)$ & $v \mathrm{C}_{2}-\mathrm{H}_{12}$ \\
\hline $\mathrm{S} 10$ & $A_{g}$ & $v \mathrm{C}-\mathrm{H}$ & $v \mathrm{C}-\mathrm{H}^{\prime}$ & $\left(v \mathrm{C}_{2}-\mathrm{H}_{12}\right)-\left(v \mathrm{C}_{3}-\mathrm{H}_{11}\right)$ & $v \mathrm{C}_{3}-\mathrm{H}_{11}$ \\
\hline $\mathrm{S} 11$ & $A_{g}^{\circ}$ & $v \mathrm{C}=\mathrm{C}$ & $v \mathrm{C}=\mathrm{C}$ & $v \mathrm{C}_{2}=\mathrm{C}_{3}$ & $v \mathrm{C}_{2}=\mathrm{C}_{3}$ \\
\hline $\mathrm{S} 12$ & $B_{u}$ & $\delta \mathrm{COH}$ a. & $\delta \mathrm{COH}$ & $\left(\delta \mathrm{C}_{1} \mathrm{O}_{6} \mathrm{H}_{7}\right)-\left(\delta \mathrm{C}_{4} \mathrm{O}_{9} \mathrm{H}_{10}\right)$ & $\delta \mathrm{C}_{1} \mathrm{O}_{6} \mathrm{H}_{7}$ \\
\hline S13 & $A_{g}$ & $\delta \mathrm{COH}$ & $\delta \mathrm{COH}^{\prime}$ & $\left(\delta \mathrm{C}_{1} \mathrm{O}_{6} \mathrm{H}_{7}\right)+\left(\delta \mathrm{C}_{4} \mathrm{O}_{9} \mathrm{H}_{10}\right)$ & $\delta \mathrm{C}_{4} \mathrm{O}_{9} \mathrm{H}_{10}$ \\
\hline $\mathrm{S} 14$ & $B_{u}^{\circ}$ & $\delta \mathrm{OCO}$ a. & $\delta \mathrm{OCO}$ & 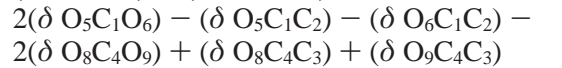 & $\begin{array}{l}2\left(\delta \mathrm{O}_{5} \mathrm{C}_{1} \mathrm{O}_{6}\right)-\left(\delta \mathrm{O}_{5} \mathrm{C}_{1} \mathrm{C}_{2}\right)- \\
\left(\delta \mathrm{O}_{6} \mathrm{C}_{1} \mathrm{C}_{2}\right)\end{array}$ \\
\hline $\mathrm{S} 15$ & $A_{g}$ & $\delta \mathrm{OCO}$ & $\delta \mathrm{OCO}^{\prime}$ & $\begin{array}{l}2\left(\delta \mathrm{O}_{5} \mathrm{C}_{1} \mathrm{O}_{6}\right)-\left(\delta \mathrm{O}_{5} \mathrm{C}_{1} \mathrm{C}_{2}\right)-\left(\delta \mathrm{O}_{6} \mathrm{C}_{1} \mathrm{C}_{2}\right)+ \\
2\left(\delta \mathrm{O}_{8} \mathrm{C}_{4} \mathrm{O}_{9}\right)-\left(\delta \mathrm{O}_{8} \mathrm{C}_{4} \mathrm{C}_{3}\right)-\left(\delta \mathrm{O}_{9} \mathrm{C}_{4} \mathrm{C}_{3}\right)\end{array}$ & $\begin{array}{l}2\left(\delta \mathrm{O}_{8} \mathrm{C}_{4} \mathrm{O}_{9}\right)-\left(\delta \mathrm{O}_{8} \mathrm{C}_{4} \mathrm{C}_{3}\right)- \\
\left(\delta \mathrm{O}_{9} \mathrm{C}_{4} \mathrm{C}_{3}\right)\end{array}$ \\
\hline S16 & $B_{u}$ & $\delta \mathrm{CC}=\mathrm{O}$ a. & $\delta \mathrm{CC}=\mathrm{O}$ & $\begin{array}{l}\left(\delta \mathrm{O}_{5} \mathrm{C}_{1} \mathrm{C}_{2}\right)-\left(\delta \mathrm{O}_{6} \mathrm{C}_{1} \mathrm{C}_{2}\right)-\left(\delta \mathrm{O}_{8} \mathrm{C}_{4} \mathrm{C}_{3}\right)+ \\
\left(\delta \mathrm{O}_{9} \mathrm{C}_{4} \mathrm{C}_{3}\right)\end{array}$ & $\left(\delta \mathrm{O}_{5} \mathrm{C}_{1} \mathrm{C}_{2}\right)-\left(\delta \mathrm{O}_{6} \mathrm{C}_{1} \mathrm{C}_{2}\right)$ \\
\hline S17 & $A_{g}$ & $\delta \mathrm{CC}=\mathrm{O}$ & $\delta \mathrm{CC}=\mathrm{O}^{\prime}$ & $\begin{array}{l}\left(\delta \mathrm{O}_{5} \mathrm{C}_{1} \mathrm{C}_{2}\right)-\left(\delta \mathrm{O}_{6} \mathrm{C}_{1} \mathrm{C}_{2}\right)+\left(\delta \mathrm{O}_{8} \mathrm{C}_{4} \mathrm{C}_{3}\right)- \\
\left(\delta \mathrm{O}_{9} \mathrm{C}_{4} \mathrm{C}_{3}\right)\end{array}$ & $\left(\delta \mathrm{O}_{8} \mathrm{C}_{4} \mathrm{C}_{3}\right)-\left(\delta \mathrm{O}_{9} \mathrm{C}_{4} \mathrm{C}_{3}\right)$ \\
\hline $\mathrm{S} 18$ & $B_{u}$ & $\delta \mathrm{CC}=\mathrm{C}$ a. & $\delta \mathrm{CC}=\mathrm{C}$ & $\begin{array}{l}2\left(\delta \mathrm{C}_{1} \mathrm{C}_{2} \mathrm{C}_{3}\right)-\left(\delta \mathrm{C}_{1} \mathrm{C}_{2} \mathrm{H}_{12}\right)-\left(\delta \mathrm{C}_{3} \mathrm{C}_{2} \mathrm{H}_{12}\right)- \\
2\left(\delta \mathrm{C}_{4} \mathrm{C}_{3} \mathrm{C}_{2}\right)+\left(\delta \mathrm{C}_{4} \mathrm{C}_{3} \mathrm{H}_{11}\right)+\left(\delta \mathrm{C}_{2} \mathrm{C}_{3} \mathrm{H}_{11}\right)\end{array}$ & $\begin{array}{l}2\left(\delta \mathrm{C}_{1} \mathrm{C}_{2} \mathrm{C}_{3}\right)-\left(\delta \mathrm{C}_{1} \mathrm{C}_{2} \mathrm{H}_{12}\right)- \\
\left(\delta \mathrm{C}_{3} \mathrm{C}_{2} \mathrm{H}_{12}\right)\end{array}$ \\
\hline S19 & $A_{g}$ & $\delta \mathrm{CC}=\mathrm{C}$ & $\delta \mathrm{CC}=\mathrm{C}^{\prime}$ & $\begin{array}{l}2\left(\delta \mathrm{C}_{1} \mathrm{C}_{2} \mathrm{C}_{3}\right)-\left(\delta \mathrm{C}_{1} \mathrm{C}_{2} \mathrm{H}_{12}\right)-\left(\delta \mathrm{C}_{3} \mathrm{C}_{2} \mathrm{H}_{12}\right)+ \\
2\left(\delta \mathrm{C}_{4} \mathrm{C}_{3} \mathrm{C}_{2}\right)-\left(\delta \mathrm{C}_{4} \mathrm{C}_{3} \mathrm{H}_{11}\right)-\left(\delta \mathrm{C}_{2} \mathrm{C}_{3} \mathrm{H}_{11}\right)\end{array}$ & $\begin{array}{l}2\left(\delta \mathrm{C}_{4} \mathrm{C}_{3} \mathrm{C}_{2}\right)-\left(\delta \mathrm{C}_{4} \mathrm{C}_{3} \mathrm{H}_{11}\right)- \\
\left(\delta \mathrm{C}_{2} \mathrm{C}_{3} \mathrm{H}_{11}\right)\end{array}$ \\
\hline $\mathrm{S} 20$ & $B_{u}$ & $\delta \mathrm{CCH}$ a. & $\delta \mathrm{CCH}$ & $\begin{array}{l}\left(\delta \mathrm{C}_{1} \mathrm{C}_{2} \mathrm{C}_{3}\right)-\left(\delta \mathrm{C}_{3} \mathrm{C}_{2} \mathrm{H}_{12}\right)-\left(\delta \mathrm{C}_{4} \mathrm{C}_{3} \mathrm{C}_{2}\right)+ \\
\left(\delta \mathrm{C}_{2} \mathrm{C}_{3} \mathrm{H}_{11}\right)\end{array}$ & $\left(\delta \mathrm{C}_{1} \mathrm{C}_{2} \mathrm{C}_{3}\right)-\left(\delta \mathrm{C}_{3} \mathrm{C}_{2} \mathrm{H}_{12}\right)$ \\
\hline $\mathrm{S} 21$ & $A_{g}$ & $\delta \mathrm{CCH}$ & $\delta \mathrm{CCH}^{\prime}$ & $\begin{array}{l}\left(\delta \mathrm{C}_{1} \mathrm{C}_{2} \mathrm{C}_{3}\right)-\left(\delta \mathrm{C}_{3} \mathrm{C}_{2} \mathrm{H}_{12}\right)+\left(\delta \mathrm{C}_{4} \mathrm{C}_{3} \mathrm{C}_{2}\right)- \\
\left(\delta \mathrm{C}_{2} \mathrm{C}_{3} \mathrm{H}_{11}\right)\end{array}$ & $\left(\delta \mathrm{C}_{4} \mathrm{C}_{3} \mathrm{C}_{2}\right)-\left(\delta \mathrm{C}_{2} \mathrm{C}_{3} \mathrm{H}_{11}\right)$ \\
\hline $\mathrm{S} 22$ & $B_{g}$ & $\gamma \mathrm{C}=\mathrm{O}$ a. & $\gamma \mathrm{C}=\mathrm{O}$ & $\left(\gamma \mathrm{C}_{1}=\mathrm{O}_{5}\right)-\left(\gamma \mathrm{C}_{4}=\mathrm{O}_{8}\right)$ & $\gamma \mathrm{C}_{1}=\mathrm{O}_{5}$ \\
\hline $\mathrm{S} 23$ & $A_{u}^{\circ}$ & $\gamma \mathrm{C}=\mathrm{O}$ & $\gamma \mathrm{C}=\mathrm{O}^{\prime}$ & $\left(\gamma \mathrm{C}_{1}=\mathrm{O}_{5}\right)+\left(\gamma \mathrm{C}_{4}=\mathrm{O}_{8}\right)$ & $\gamma \mathrm{C}_{4}=\mathrm{O}_{8}$ \\
\hline $\mathrm{S} 24$ & $B_{g}$ & $\gamma \mathrm{CH}$ a. & $\gamma \mathrm{CH}$ & $\left(\gamma \mathrm{C}_{2} \mathrm{H}_{12}\right)-\left(\gamma \mathrm{C}_{3} \mathrm{H}_{11}\right)$ & $\gamma \mathrm{C}_{2} \mathrm{H}_{12}$ \\
\hline $\mathrm{S} 25$ & $A_{u}^{\circ}$ & $\gamma \mathrm{CH}$ & $\gamma \mathrm{CH}^{\prime}$ & $\left(\gamma \mathrm{C}_{2} \mathrm{H}_{12}\right)+\left(\gamma \mathrm{C}_{3} \mathrm{H}_{11}\right)$ & $\gamma \mathrm{C}_{3} \mathrm{H}_{11}$ \\
\hline S26 & $B_{g}$ & $\tau \mathrm{C}-\mathrm{O}$ a. & $\tau \mathrm{C}-\mathrm{O}$ & $\left(\tau \mathrm{C}_{1}-\mathrm{O}_{6}\right)-\left(\tau \mathrm{C}_{4}-\mathrm{O}_{9}\right)$ & $\tau \mathrm{C}_{1}-\mathrm{O}_{6}$ \\
\hline S27 & $A_{u}^{\circ}$ & $\tau \mathrm{C}-\mathrm{O}$ & $\tau \mathrm{C}-\mathrm{O}^{\prime}$ & $\left(\tau \mathrm{C}_{1}-\mathrm{O}_{6}\right)+\left(\tau \mathrm{C}_{4}-\mathrm{O}_{9}\right)$ & $\tau \mathrm{C}_{4}-\mathrm{O}_{9}$ \\
\hline $\mathrm{S} 28$ & $B_{g}$ & $\tau \mathrm{C}-\mathrm{C}$ a. & $\tau \mathrm{C}-\mathrm{C}$ & $\left(\tau \mathrm{C}_{1}-\mathrm{C}_{2}\right)-\left(\tau \mathrm{C}_{3}-\mathrm{C}_{4}\right)$ & $\tau \mathrm{C}_{1}-\mathrm{C}_{2}$ \\
\hline S29 & $A_{u}$ & $\tau \mathrm{C}-\mathrm{C}$ & $\tau \mathrm{C}-\mathrm{C}^{\prime}$ & $\left(\tau \mathrm{C}_{1}-\mathrm{C}_{2}\right)+\left(\tau \mathrm{C}_{3}-\mathrm{C}_{4}\right)$ & $\tau \mathrm{C}_{3}-\mathrm{C}_{4}$ \\
\hline $\mathrm{S} 30$ & $A_{u}$ & $\tau \mathrm{C}=\mathrm{C}$ & $\tau \mathrm{C}=\mathrm{C}$ & $\tau \mathrm{C}_{2}=\mathrm{C}_{3}$ & $\tau \mathrm{C}_{2}=\mathrm{C}_{3}$ \\
\hline
\end{tabular}

${ }^{a}$ Symmetry species refer to the higher symmetry conformer $\left(C_{2 h}\right) .{ }^{b}$ Used in the normal coordinate analysis of conformers I and II of maleic acid and form II of fumaric acid. ${ }^{c}$ Used in the normal coordinate analysis of forms I and III of fumaric acid. ${ }^{c}$ a., asymmetric; $v$, stretching; $\delta$, bending; $\gamma$, rocking; $\tau$, torsion, ', $\mathrm{O}_{8}=\mathrm{C}_{4}\left(-\mathrm{O}_{9}-\mathrm{H}_{10}\right)-\mathrm{C}_{3}\left(-\mathrm{H}_{12}\right)$ fragment. ${ }^{d}$ For atom numbering see Figures 1 and 2.

and B3LYP) lead to qualitatively similar results. All methods predict three planar conformers (I, II, and III in Figure 2), which have relative energies within $5 \mathrm{~kJ} \mathrm{~mol}^{-1}$ and two conformers (IV and $\mathrm{V}$ in Figure 2) ca. $25 \mathrm{~kJ} \mathrm{~mol}^{-1}$ above these three planar structures. The planar conformers exhibit cis arrangements around the $\mathrm{C}-\mathrm{O}$ bonds, differing in the conformation assumed by their $\mathrm{O}=\mathrm{C}-\mathrm{C}=\mathrm{C}$ axes (see Figure 2). Conformers I and III belong to the $C_{2 h}$ symmetry point group and they are characterized by two s-cis or two s-trans $\mathrm{O}=\mathrm{C}-\mathrm{C}=\mathrm{C}$ axes, respectively. Conformer II $\left(\mathrm{C}_{\mathrm{s}}\right)$ has one s-cis and one s-trans $\mathrm{O}=\mathrm{C}-\mathrm{C}=\mathrm{C}$ axis. Hence, in consonance with the previously reported relative energies of the s-cis and s-trans arrangements around the $\mathrm{C}_{\alpha}-\mathrm{C}$ bond in $\alpha, \beta$-unsaturated carboxylic acids, ${ }^{13-15}$ the relative energy of the three most stable conformers of fumaric acid increases with the number of s-trans $\mathrm{O}=\mathrm{C}-\mathrm{C}=\mathrm{C}$ axes. This correlates with the existence of attractive interactions between the oxygen atoms of the carboxylic groups and the $\beta$-hydrogen atoms (weak $\mathrm{CH} \cdots \mathrm{O}$ hydrogen bond) that are important in determining the relative energy of the three most stable conformers. ${ }^{13-15}$ In each of the three most stable conformers of fumaric acid, two $\mathrm{O} \cdots \beta$-hydrogen attractions are found, as shown in Figure 2. However, this interaction is stronger when it involves the carbonyl oxygen, as this atom shows a higher ability to act as an $\mathrm{H}$-bond acceptor than the hydroxylic oxygen atom. ${ }^{28}$ The different strengths of the $\mathrm{CH} \cdots \mathrm{O}=\mathrm{C}$ and $\mathrm{CH} \cdot \cdots \mathrm{OH}$ interactions may hence be used to justify the energy differences between conformers I, II, and III, since there are two $\mathrm{C}-\mathrm{H} \cdots \mathrm{O}=\mathrm{C}$ interactions present in conformer I but only one in conformer II and none in conformer III.

Conformers IV and V are similar to conformers I and II but have one trans carboxylic group $\left(\mathrm{O}=\mathrm{C}-\mathrm{O}-\mathrm{H}\right.$ dihedral of $180^{\circ}$; see Figure 2); they are ca. $25-30 \mathrm{~kJ} \mathrm{~mol}^{-1}$ above the global minimum, structure I. Besides the general higher intrinsic energy associated with a trans $\mathrm{O}=\mathrm{C}-\mathrm{O}-\mathrm{H}$ axis relative to a cis $\mathrm{O}=\mathrm{C}-\mathrm{O}-\mathrm{H}$ axis, ${ }^{27}$ in fumaric acid a trans arrangement of this axis leads to the appearance of a steric repulsion between the hydrogen atom of the trans carboxylic group and the corresponding $\alpha$-hydrogen atom. These two factors account for the high energy of conformers IV and V. Note that the conformation analogous to conformer III but having one of its carboxylic groups in the trans conformation was not found to be a minimum, whichever the level of theory considered. A similar situation was observed by changing the conformation of the second carboxylic group in structure II. This can be explained considering that, in these cases, the molecule is strongly destabilized by the presence of strong $\mathrm{H} \cdots \mathrm{H}$ repulsive 
TABLE 4: Observed (Ar matrix) and Calculated B3LYP/6-31G(d,p) Vibrational Frequencies and IR Intensities ${ }^{a}$ of the Most Stable Conformer of Maleic Acid

\begin{tabular}{|c|c|c|c|c|c|}
\hline \multicolumn{6}{|c|}{ Bands Increasing in Intensity upon NIR Irradiation and Annealing of the Matrix Assigned to Conformer I } \\
\hline $\begin{array}{l}\text { approximate } \\
\text { description }\end{array}$ & $v_{\exp }^{b}$ & $I_{\text {exp }}$ & $v_{\text {calc }}$ & $I_{\text {calc }}$ & $\mathrm{PED}^{c}$ \\
\hline$v \mathrm{OH}^{\prime}$ & 3550 & 29 & 3610 & 22 & $v \mathrm{OH}^{\prime}(100)$ \\
\hline$v \mathrm{OH}$ & 3110 & 52 & 3210 & 137 & $v \mathrm{OH}(100)$ \\
\hline$v \mathrm{CH}$ & n.o. & & 3088 & $<1$ & $v \mathrm{CH}(55)+v \mathrm{CH}^{\prime}(44)$ \\
\hline \multirow[t]{3}{*}{$v \mathrm{CH}^{\prime}$} & n.o. & & 3072 & $<1$ & $v \mathrm{CH}^{\prime}(54)+v \mathrm{CH}(44)$ \\
\hline & 2784 & 9 & & & $2 \times 1410^{d}$ \\
\hline & 2770 & 4 & & & $2 \times 1403^{d}$ \\
\hline \multirow[t]{3}{*}{$v \mathrm{C}=\mathrm{O}$} & $\int 1762$ & 15 & 1754 & 44 & $v \mathrm{C}=\mathrm{O}(63)$ \\
\hline & 1750 & 21 & & & \\
\hline & 1740 & 4 & & & \\
\hline \multirow[t]{2}{*}{$v \mathrm{C}=\mathrm{O}^{\prime}$} & 1722 & 35 & 1699 & 67 & $v \mathrm{C}=\mathrm{O}^{\prime}(62)$ \\
\hline & 1712 & 9 & & & $2 \times 857^{d}$ \\
\hline \multirow[t]{2}{*}{$v \mathrm{C}=\mathrm{C}$} & 1635 & 26 & 1629 & 20 & $v \mathrm{C}=\mathrm{C}(66)$ \\
\hline & 1568 & 6 & & & $1202+374^{d}$ \\
\hline \multirow[t]{2}{*}{$\delta \mathrm{COH}$} & $\{1428$ & 36 & 1405 & 46 & $\delta \mathrm{COH}(68)$ \\
\hline & 1420 & 47 & & & \\
\hline \multirow[t]{4}{*}{$\delta \mathrm{CCH}^{\prime}$} & $\int 1410$ & 13 & 1396 & 27 & $\delta \mathrm{CCH}^{\prime}(26)$ \\
\hline & 1406 & 7 & & & \\
\hline & 1403 & 43 & & & \\
\hline & 1369 & 4 & & & $606+767^{d}$ \\
\hline$\delta \mathrm{COH}^{\prime}$ & 1313 & 8 & 1296 & 6 & $\delta \mathrm{COH}^{\prime}(23)+\delta \mathrm{CCH}(22)$ \\
\hline \multirow{2}{*}{$v \mathrm{C}-\mathrm{O}$} & 1296 & 2 & 1276 & 2 & $v \mathrm{C}-\mathrm{O}(32)$ \\
\hline & 1246 & 13 & & & $2 \times 631^{d}$ \\
\hline \multirow[t]{2}{*}{$\delta \mathrm{CCH}$} & 1202 & 8 & & 8 & $589+606^{d}$ \\
\hline & 1174 & 26 & 1187 & & $\delta \mathrm{CCH}(17)+\delta \mathrm{CCH}^{\prime}(23)+v \mathrm{C}-\mathrm{O}(17)$ \\
\hline \multirow[t]{3}{*}{$v \mathrm{C}-\mathrm{O}^{\prime}$} & $\{1154$ & 7 & 1147 & 82 & $v \mathrm{C}-\mathrm{O}^{\prime}(35)+\delta \mathrm{COH}^{\prime}(36)$ \\
\hline & 1152 & 66 & & & \\
\hline & 1138 & 7 & & & $589+552^{d}$ \\
\hline \multirow[t]{2}{*}{$\gamma \mathrm{CH}$} & $\{1025$ & 2 & 993 & $<1$ & $\gamma \mathrm{CH}(53)+\gamma \mathrm{CH}^{\prime}(32)+\tau \mathrm{C}=\mathrm{C}(23)$ \\
\hline & 1027 & $<1$ & & & \\
\hline$v \mathrm{C}-\mathrm{C}^{\prime}$ & 933 & $<1$ & 915 & 4 & $v \mathrm{C}-\mathrm{C}^{\prime}(19)+\delta \mathrm{CCC}^{\prime}(32)+\delta \mathrm{CCC}(23)$ \\
\hline$\gamma \mathrm{CH}^{\prime}$ & 857 & 15 & 830 & 8 & $\gamma \mathrm{CH}^{\prime}(41)+\gamma \mathrm{CH}(24)$ \\
\hline$\tau \mathrm{C}-\mathrm{O}$ & 822 & 35 & 789 & 23 & $\tau \mathrm{C}-\mathrm{O}(46)+\tau \mathrm{C}=\mathrm{C}(18)$ \\
\hline$v \mathrm{C}-\mathrm{O}$ & 782 & 3 & 806 & $<1$ & $v \mathrm{C}-\mathrm{C}(26)+\delta$ OCO $(19)$ \\
\hline$\delta \mathrm{CCC}$ & 775 & 3 & 746 & 2 & $\delta \operatorname{CCC}(31)+\delta \operatorname{CCC}^{\prime}(27)$ \\
\hline$\gamma \mathrm{C}=\mathrm{O}^{\prime}$ & 767 & 3 & 737 & 2 & $\gamma \mathrm{C}=\mathrm{O}^{\prime}(18)+\tau \mathrm{C}=\mathrm{C}(29)$ \\
\hline$\tau \mathrm{C}-\mathrm{O}^{\prime}$ & 631 & 34 & 640 & 25 & $\tau \mathrm{C}-\mathrm{O}^{\prime}(57)+\tau \mathrm{C}=\mathrm{C}(17)$ \\
\hline$\delta \mathrm{OCO}^{\prime}$ & 606 & 16 & 584 & 7 & $\delta \mathrm{OCO}^{\prime}(26)+\delta \mathrm{OCO}(20)+\delta \mathrm{CCC}(14)$ \\
\hline$\delta \mathrm{OCO}$ & 589 & 19 & 568 & 9 & $\delta \mathrm{OCO}(33)+\delta \mathrm{OCO}^{\prime}(29)$ \\
\hline$\gamma \mathrm{C}=\mathrm{O}$ & 552 & 3 & 544 & 3 & $\gamma \mathrm{C}=\mathrm{O}(23)+\gamma \mathrm{C}=\mathrm{O}^{\prime}(24)+\gamma \mathrm{CH}$ (17) \\
\hline$\delta \mathrm{CC}=\mathrm{O}$ & & & 374 & $<1$ & $\delta \mathrm{CC}=\mathrm{O}(38)+\delta \mathrm{CC}=\mathrm{O}^{\prime}(27)$ \\
\hline$\delta \mathrm{CC}=\mathrm{O}^{\prime}$ & & & 280 & 2 & $\delta \mathrm{CC}=\mathrm{O}^{\prime}(15)+\delta \mathrm{CCC}(38)+\delta \mathrm{CCC}^{\prime}(22)$ \\
\hline$\tau \mathrm{C}=\mathrm{C}$ & & & 271 & 2 & $\tau \mathrm{C}=\mathrm{C}(60)+\tau \mathrm{C}-\mathrm{C}(18)$ \\
\hline$\delta \mathrm{CCC}^{\prime}$ & & & 234 & 1 & $\delta \operatorname{CCC}^{\prime}(59)$ \\
\hline$\tau \mathrm{C}-\mathrm{C}^{\prime}$ & & & 88 & $<1$ & $\tau \mathrm{C}-\mathrm{C}^{\prime}(48)+\tau \mathrm{C}-\mathrm{C}(45)$ \\
\hline$\tau \mathrm{C}-\mathrm{C}$ & & & 46 & $<1$ & $\tau \mathrm{C}-\mathrm{C}(63)$ \\
\hline
\end{tabular}

${ }^{a}$ Frequencies in $\mathrm{cm}^{-1}$; intensities in $\mathrm{km} \mathrm{mol}^{-1}$; calculated frequencies were scaled by 0.9614 ; experimental intensity values presented correspond to the observed integrated intensities of each conformer normalized to 1000. Calculated intensities were scaled to experimental integrated normalized intensities (only bands which have an experimental counterpart and can be ascribable to a single conformer were used in the scaling procedure). ${ }^{b}$ Frequency values shown in bold represent bands assigned to more than one conformer. In these case, the experimental intensities shall be compared with the sum of the calculated intensities for the two observed conformers (see also Table 5). ${ }^{c}$ Only contributions higher than $15 \%$ are listed. ${ }^{d}$ Tentative assignment as nonfundamental modes (overtones and combination bands). $v$, stretching; $\delta$, bending; $\tau$, torsion; $\gamma$, rocking, n.o., non observed; ', refers to $\mathrm{O}_{8}=\mathrm{C}_{4}\left(-\mathrm{O}_{9}-\mathrm{H}_{10}\right)-\mathrm{C}_{3}\left(-\mathrm{H}_{12}\right)$ fragment. Minor bands due to nonfundamental modes where also observed at 2686, 1573, 1567, 1540,1537 , and $1454 \mathrm{~cm}^{-1}$.

interactions between the hydrogen atom of the trans carboxylic group and the $\beta$-hydrogen atom. On the other hand, the conformation analogous to conformer I with both carboxylic groups trans, was predicted to have an energy that is nearly twice that calculated for the structure showing only one trans carboxylic group (this conformation was predicted to be a minimum on the B3LYP PES, while it was found to correspond to a saddle point on the MP2 PES).

In Table 2 we present the geometrical parameters of the three most stable conformers of fumaric acid. In agreement with our previous studies on $\alpha, \beta$-unsaturated carboxylic acids, ${ }^{13,15}$ the present calculations show that a s-trans $\mathrm{O}=\mathrm{C}-\mathrm{C}=\mathrm{C}$ axis favors the $\pi$ electron delocalization in the molecule. This effect reflects the dependence of the $\mathrm{C}_{\alpha}-\mathrm{C}$ and $\mathrm{C}=\mathrm{C}$ bond lengths on the conformation of the $\mathrm{O}=\mathrm{C}-\mathrm{C}=\mathrm{C}$ axis. The $\mathrm{C}_{\alpha}-\mathrm{C}$ bond length decreases and the $\mathrm{C}=\mathrm{C}$ bond elongates slightly for a s-trans conformation compared to the situation in conformer I. For instance, the $\mathrm{C}_{\alpha}-\mathrm{C}$ and $\mathrm{C}=\mathrm{C}$ bond lengths are predicted by the MP2 calculations to be 147.9 and $134.3 \mathrm{pm}$, respectively, in conformer III, while in the most stable conformer these bond lengths are 148.2 and $134.2 \mathrm{pm}$.

Matrix-Isolation Infrared Studies. The infrared spectrum of isolated maleic and fumaric acid in solid argon clearly reveals the presence several conformers. A detailed assignment of the spectra was undertaken by comparison with previous studies of other $\alpha, \beta$-unsaturated carboxylic acids ${ }^{13-15}$ and with the 
TABLE 5: Observed (Ar matrix) and Calculated B3LYP/6-31G(d,p) Vibrational Frequencies and IR Intensities ${ }^{a}$ of Conformer II of Maleic Acid

\begin{tabular}{|c|c|c|c|c|c|}
\hline \multicolumn{6}{|c|}{ Bands Decreasing in Intensity upon NIR Irradiation and Annealing of the Matrix Assigned to Conformer II } \\
\hline $\begin{array}{l}\text { approximate } \\
\text { description }\end{array}$ & $v_{\text {exp }}^{b}$ & $I_{\text {exp }}$ & $v_{\text {calc }}$ & $I_{\text {calc }}$ & $\mathrm{PED}^{c}$ \\
\hline$v \mathrm{OH}^{\prime}$ & 3566 & 6 & 3616 & 14 & $v \mathrm{OH}^{\prime}(100)$ \\
\hline$v \mathrm{OH}$ & 3561 & 11 & 3600 & 13 & $v \mathrm{OH}(100)$ \\
\hline$v \mathrm{CH}^{\prime}$ & n.o. & & 3091 & $<1$ & $v \mathrm{CH}^{\prime}(88)$ \\
\hline \multirow[t]{2}{*}{$v \mathrm{CH}$} & n.o. & & 3066 & $<1$ & $v \mathrm{CH}(88)$ \\
\hline & 1808 & 1 & & & \\
\hline \multirow[t]{7}{*}{$v \mathrm{C}=\mathrm{O}$} & 1780 & 5 & 1772 & 53 & $v \mathrm{C}=\mathrm{O}(78)$ \\
\hline & 1796 & 1 & & & \\
\hline & 1790 & 5 & & & \\
\hline & 1783 & 4 & & & \\
\hline & 1778 & 11 & & & \\
\hline & 1773 & 13 & & & \\
\hline & 1769 & 9 & & & \\
\hline \multirow[t]{2}{*}{$v \mathrm{C}=\mathrm{O}^{\prime}$} & $\{1761$ & 13 & 1756 & 45 & $v \mathrm{C}=\mathrm{O}^{\prime}(77)$ \\
\hline & 1754 & 4 & & & \\
\hline \multirow[t]{2}{*}{$v \mathrm{C}=\mathrm{C}$} & 1698 & 6 & 1649 & 12 & $v \mathrm{C}=\mathrm{C}(71)$ \\
\hline & 1570 & $<1$ & & & $1122+448^{d}$ \\
\hline$\delta \mathrm{CCH}^{\prime}$ & 1420 & 47 & 1394 & 47 & $\delta \mathrm{CCH}^{\prime}(29)+\delta \mathrm{CCH}(17)$ \\
\hline$v \mathrm{C}-\mathrm{O}$ & 1370 & 7 & 1328 & 8 & $v \mathrm{C}-\mathrm{O}(23)+\delta \mathrm{COH}(22)$ \\
\hline$\delta \mathrm{COH}^{\prime}$ & 1290 & 3 & 1279 & 3 & $\delta \mathrm{COH}^{\prime}(29)+\delta \mathrm{CCH}(24)$ \\
\hline$\delta \mathrm{CCH}$ & 1163 & 4 & 1179 & 9 & $\delta \mathrm{CCH}(39)+\delta \mathrm{CCH}^{\prime}(26)$ \\
\hline \multirow[t]{2}{*}{$\delta \mathrm{COH}$} & $\{1150$ & 33 & 1148 & 44 & $\delta \mathrm{COH}(29)+v \mathrm{C}-\mathrm{O}(24)$ \\
\hline & 1147 & 8 & & & \\
\hline$v \mathrm{C}-\mathrm{O}^{\prime}$ & 1122 & 66 & 1114 & 77 & $v \mathrm{C}-\mathrm{O}^{\prime}(30)+\delta \mathrm{COH}^{\prime}(21)$ \\
\hline$\gamma \mathrm{CH}^{\prime}$ & n.o. & & 958 & $<1$ & $\gamma \mathrm{CH}^{\prime}(49)+\gamma \mathrm{CH}(38)$ \\
\hline$v \mathrm{C}-\mathrm{C}^{\prime}$ & 934 & 7 & 926 & 1 & $v \mathrm{C}-\mathrm{C}^{\prime}(21)+\delta \mathrm{CCC}^{\prime}(19)+\delta \mathrm{CCC}(19)$ \\
\hline$v \mathrm{C}-\mathrm{C}$ & 828 & 5 & 809 & 3 & $v \mathrm{C}-\mathrm{C}(20)+v \mathrm{C}-\mathrm{C}^{\prime}(25)$ \\
\hline$\gamma \mathrm{C}=\mathrm{O}^{\prime}$ & 811 & 11 & 792 & 10 & $\gamma \mathrm{C}=\mathrm{O}^{\prime}(29)+\gamma \mathrm{CH}^{\prime}(31)+\gamma \mathrm{C}=\mathrm{O}(17)$ \\
\hline$\gamma \mathrm{C}=\mathrm{O}$ & 781 & 3 & 762 & 7 & $\gamma \mathrm{C}=\mathrm{O}(26)$ \\
\hline$\gamma \mathrm{CH}$ & n.o. & & 718 & 3 & $\gamma \mathrm{CH}(23)+\tau \mathrm{C}=\mathrm{C}(21)+\gamma \mathrm{C}=\mathrm{O}^{\prime}(19)$ \\
\hline$\tau \mathrm{C}-\mathrm{O}$ & 631 & 34 & 645 & 10 & $\tau \mathrm{C}-\mathrm{O}(44)$ \\
\hline$\tau \mathrm{C}-\mathrm{O}^{\prime}$ & 591 & 8 & 607 & 13 & $\tau \mathrm{C}-\mathrm{O}^{\prime}(54)+\tau \mathrm{C}=\mathrm{C}(23)$ \\
\hline$\delta \mathrm{OCO}^{\prime}$ & 568 & 13 & 567 & 20 & $\delta \mathrm{OCO}^{\prime}(33)+\tau \mathrm{C}-\mathrm{O}(30)$ \\
\hline$\delta \mathrm{OCO}$ & 549 & 23 & 532 & 13 & $\delta \mathrm{OCO}(59))$ \\
\hline$\delta \mathrm{CCC}^{\prime}$ & n.o. & & 451 & 2 & $\delta \mathrm{CCC}^{\prime}(15)+\gamma \mathrm{C}=\mathrm{O}(24)+\delta \mathrm{OCO}^{\prime}(19)$ \\
\hline$\delta \mathrm{CC}=\mathrm{O}$ & n.o. & & 425 & 3 & $\delta \mathrm{CC}=\mathrm{O}(24)+\tau \mathrm{C}=\mathrm{C}(27)+\gamma \mathrm{CH}^{\prime}(24)$ \\
\hline$\delta \mathrm{CC}=\mathrm{O}^{\prime}$ & & & 272 & $<1$ & $\delta \mathrm{CC}=\mathrm{O}^{\prime}(34)+\delta \mathrm{CCC}(20)$ \\
\hline$\tau \mathrm{C}=\mathrm{C}$ & & & 224 & $<1$ & $\tau \mathrm{C}=\mathrm{C}(58)$ \\
\hline$\delta \mathrm{CCC}$ & & & 135 & $<1$ & $\delta \mathrm{CCC}(43)+\delta \mathrm{CCC}^{\prime}(33)$ \\
\hline$\tau \mathrm{C}-\mathrm{C}^{\prime}$ & & & 78 & $<1$ & $\tau \mathrm{C}-\mathrm{C}^{\prime}(26)+\tau \mathrm{C}-\mathrm{C}(17)+\delta \mathrm{CCC}^{\prime}(17)$ \\
\hline$\tau \mathrm{C}-\mathrm{C}$ & & & 33 & $<1$ & $\tau \mathrm{C}-\mathrm{C}(64)$ \\
\hline
\end{tabular}

${ }^{a}$ Frequencies in $\mathrm{cm}^{-1}$; intensities in $\mathrm{km} \mathrm{mol}^{-1}$; calculated frequencies were scaled by 0.9614 ; experimental intensity values presented correspond to the observed integrated intensities of each conformer normalized to 1000. Calculated intensities were scaled to experimental integrated normalized intensities (only bands which have an experimental counterpart and can be ascribable to a single conformer were used in the scaling procedure). ${ }^{b}$ Frequency values shown in bold represent bands assigned to more than one conformer. In these case, the experimental intensities shall be compared with the sum of the calculated intensities for the two observed conformers (see also Table 4). ${ }^{c}$ Only contributions higher than $15 \%$ are listed. ${ }^{d}$ Tentative assignment as combination band. $v$, stretching; $\delta$, bending; $\tau$, torsion; $\gamma$, rocking, n.o., non observed; ', refers to $\mathrm{O}_{8}=\mathrm{C}_{4}\left(-\mathrm{O}_{9}-\mathrm{H}_{10}\right)-$ $\mathrm{C}_{3}\left(-\mathrm{H}_{12}\right)$ fragment. Minor bands due to nonfundamental modes where also observed at 2686, 1568, 1539, 1343, 1341 , and $1186 \mathrm{~cm}^{-1}$.

calculated spectra (B3LYP). Besides, results from annealing experiments and in situ near-infrared (NIR) irradiation of the matrix sample showing conformational isomerization of the studied molecules were also used to assign the experimentally observed absorptions.

Table 3 presents the symmetry coordinates used to perform the normal coordinate analysis. We have used two different sets of symmetry coordinates, one for the conformers of maleic and fumaric acid belonging either to the $C_{s}$ or $C_{1}$ symmetry point groups and another for the $C_{2 h}$ conformers of fumaric acid.

Maleic Acid. The experimental and calculated wavenumbers and intensities as well as potential energy distribution (PED) for the observed absorption bands of maleic acid are collected in Tables 4 and 5. For maleic acid we were able to promote conformer isomerization both by NIR irradiation and annealing of the matrix. Therefore, the assignment of the bands of the various conformers of maleic acid is rather straightforward: the increasing bands upon NIR irradiation and annealing at $35 \mathrm{~K}$ are ascribable to the lowest energy conformer (conformer I) and the decreasing bands belong to the second most stable conformer predicted by the calculations (conformer II). No evidence was found of presence of other conformers.

The IR spectrum of matrix isolated maleic acid is shown in Figure 3. In the $\mathrm{OH}$ stretching region, the spectrum shows bands at 3566,3560 , and $3550 \mathrm{~cm}^{-1}$, which are typical values for an $\mathrm{O}-\mathrm{H}$ group not involved in hydrogen bonding (see Figure $3 \mathrm{a}$ ). The intense band at $\sim 3110 \mathrm{~cm}^{-1}$, can be assigned to an $\mathrm{O}-\mathrm{H}$ group participating in a strong hydrogen bond. According to the calculations, we assign the higher frequency bands to conformer II. The bands at 3550 and $3110 \mathrm{~cm}^{-1}$ are due to the vibrations of the free and intramolecularly hydrogen bonded $\mathrm{OH}$ group of the most stable conformer, respectively.

In the $\mathrm{C}=\mathrm{O}$ stretching region, a complex multiplet of bands appears in the $1808-1754 \mathrm{~cm}^{-1}$ region, which are due to carbonyl groups not involved in hydrogen bonding. Besides this multiplet, an intense carbonyl band is found at $1722 \mathrm{~cm}^{-1}$, and 


\section{$\mathbf{a}$}
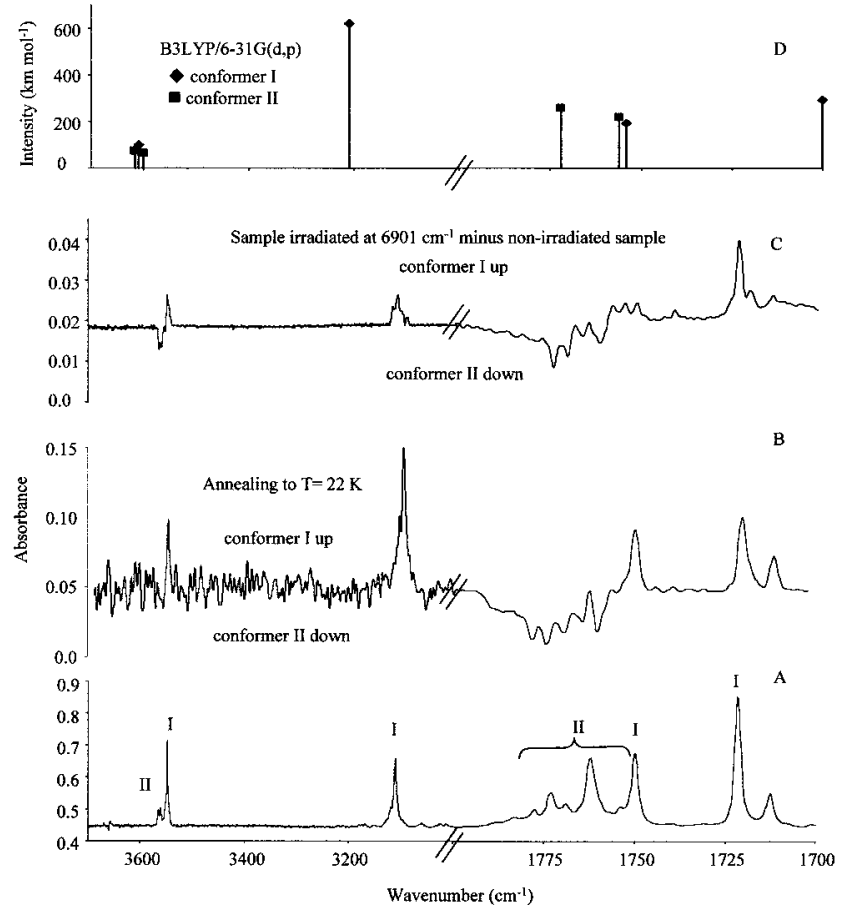
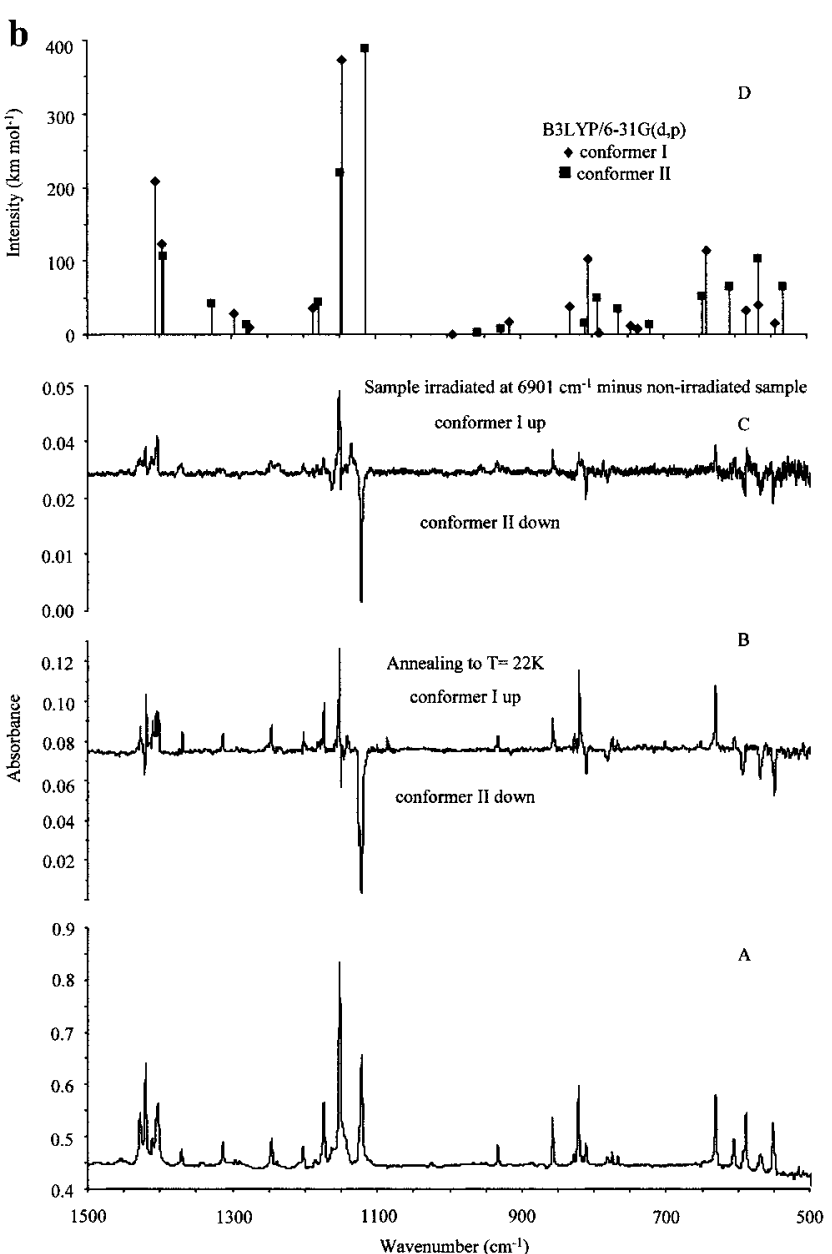

Figure 3. IR spectra of maleic acid [(a) $3600-3000 \mathrm{~cm}^{-1}$ region, (b) $1500-500 \mathrm{~cm}^{-1}$ region], (A) Ar matrix ( $T=7.5 \mathrm{~K}$ ); (B) difference spectrum (spectrum at $T=22 \mathrm{~K}$ minus spectrum at $T=9 \mathrm{~K}$ ); (C) difference spectrum (irradiated matrix at $6901 \mathrm{~cm}^{-1}$ minus nonirradiated sample); (D) calculated spectra (B3LYP).

it is red shifted (due to hydrogen bonding) compared with the unperturbed carbonyl absorptions. Taking into consideration the theoretical results, the latter band is assigned to the $\mathrm{C}=\mathrm{O}$ stretching mode of the hydrogen bonded carbonyl of conformer I, while the different components are ascribed to the vibrations originated from the non-hydrogen-bonded carbonyl of conformers I and II.

As shown in Figure 3b, the results obtained for other spectral regions fully agree with the discussion above made for the $\mathrm{OH}$ and $\mathrm{C}=\mathrm{O}$ stretching regions. For example, the most intense band in the spectrum, which appears as a doublet at 1154 and 1152 $\mathrm{cm}^{-1}$ is ascribed to the $v \mathrm{C}-\mathrm{O}^{\prime}$ mode in conformer $\mathrm{I}$ that is predicted by the calculations at $1148 \mathrm{~cm}^{-1}$. In the difference spectra showing the results of irradiation and annealing ( $B$ and $\mathrm{C}$ in Figure 3b) it is clear that, overlapped with the $1152 \mathrm{~cm}^{-1}$ band, a doublet appears with frequency maxima at 1150 and $1147 \mathrm{~cm}^{-1}$ which is due to conformer II and is assigned to $\delta$ $\mathrm{COH}$ of this form (calculated value, $1148 \mathrm{~cm}^{-1}$ ). The $v \mathrm{C}-\mathrm{O}^{\prime}$ mode in conformer II is predicted at $1114 \mathrm{~cm}^{-1}$ and is easily assigned to the band at $1122 \mathrm{~cm}^{-1}$ that decreases in intensity upon irradiation and after annealing. In turn, $\delta \mathrm{COH}$ in conformer I (calculated value: $1405 \mathrm{~cm}^{-1}$ ) gives rise to a doublet at 1428 and $1420 \mathrm{~cm}^{-1}$ (the low-frequency component is superimposed with the $\delta \mathrm{CCH}^{\prime}$ mode of conformer IIcalculated value, $1394 \mathrm{~cm}^{-1}$-as noticed in the difference spectra).

The bands ascribed to $v \mathrm{C}-\mathrm{O}$ and $\delta \mathrm{COH}^{\prime}$ are considerably less intense (see Tables 4 and 5) than those due to $v \mathrm{C}-\mathrm{O}^{\prime}$ and $\delta \mathrm{COH}$ but, nevertheless, their assignment was not difficult since they lie in less congested spectral regions.

The bands associated with vibrations involving mainly bonds with large dipole moments, such as the $\mathrm{C}=\mathrm{O}$ and $\mathrm{C}-\mathrm{O}$ stretching modes and the $\mathrm{COH}$ bendings, appear site-splitted in the matrix experiments. This fact is particularly evident for the vibrations of conformer II, where 10 component bands are ascribable to the $\mathrm{C}=\mathrm{O}$ stretching modes.

Fumaric Acid. The assignment of the infrared spectrum of fumaric acid is presented in Tables 6 and 7. For this molecule, the assignments were made basically taking into consideration the calculated spectra for the three most stable conformers, since either annealing or irradiation (both narrowband tunable NIR and broadband IR irradiation) of the matrix did not lead to any observable changes in the spectrum. Thus, the proposed assignments for fumaric acid have a higher uncertainty than those presented for maleic acid, especially for bands appearing in more congested spectral regions. Moreover, the structure of the three low-energy conformers of fumaric acid is relatively similar (e.g., all carboxylic groups are in the cis conformation), leading to similar frequencies for the various conformers hence making their detailed assignment more difficult.

The presence of conformer II in the matrix is unequivocal, since this is a conformer of fumaric acid that has no symmetry restrictions for observation of any fundamental vibrations. Thus, for instance, the band observed at $1650 \mathrm{~cm}^{-1}$, which is a characteristic absorption band of a $\mathrm{C}=\mathrm{C}$ stretching mode, should be assigned to this conformer, since this vibration $\left(A_{g}\right)$ is IR- 
TABLE 6: Observed (Ar matrix) and Calculated B3LYP/6-31G(d,p) Vibrational Frequencies and IR Intensities ${ }^{a}$ of Conformers I and III of Fumaric Acid ( $C_{2 h}$ Symmetry)

\begin{tabular}{|c|c|c|c|c|c|c|c|c|c|c|}
\hline \multirow{2}{*}{$\begin{array}{l}\text { approximate } \\
\text { description }\end{array}$} & \multicolumn{5}{|r|}{ conformer I } & \multicolumn{5}{|r|}{ conformer III } \\
\hline & $v_{\exp ^{b}}^{b}$ & $I_{\text {exp }}$ & $v_{\text {calc }}$ & $I_{\text {calc }}$ & $\mathrm{PED}^{c}$ & $v_{\exp }^{b}$ & $I_{\text {exp }}$ & $v_{\text {calc }}$ & $I_{\text {calc }}$ & $\mathrm{PED}^{c}$ \\
\hline$v \mathrm{O}-\mathrm{H}$ a. & 3560 & 54 & 3611 & 31 & $v \mathrm{O}-\mathrm{H}$ a. $(100)$ & 3576 & 22 & 3620 & 19 & $v \mathrm{O}-\mathrm{H}$ a. $(100)$ \\
\hline$v \mathrm{CH}$ a. & 2948 & 27 & 3099 & $<1$ & $v \mathrm{CH}$ a. $(100)$ & 2948 & 27 & 3113 & $<1$ & $v \mathrm{CH}$ a. $(100)$ \\
\hline$v \mathrm{C}=\mathrm{O}$ a. & 1765 & 16 & 1750 & 97 & $v \mathrm{C}=\mathrm{O}$ a. $(78)$ & 1765 & 16 & 1754 & 74 & $v \mathrm{C}=\mathrm{O}$ a. $(79)$ \\
\hline & 1763 & 15 & & & & 1763 & 15 & & & \\
\hline & 1759 & 29 & & & & 1759 & 29 & & & \\
\hline & 1756 & 84 & & & & 1756 & 84 & & & \\
\hline & 1754 & 38 & & & & 1754 & 38 & & & \\
\hline & 1750 & 39 & & & & 1750 & 39 & & & \\
\hline & & & & & & 1388 & 4 & & & $1256+133^{d}$ \\
\hline$v \mathrm{C}-\mathrm{O}$ a. & 1369 & 61 & 1352 & 71 & $\begin{array}{l}v \mathrm{C}-\mathrm{O} \text { a. }(27)+\delta \mathrm{COH} \text { a. }(27)+ \\
\qquad v \mathrm{C}-\mathrm{Ca} \text {. }(22)\end{array}$ & $\left\{\begin{array}{l}1315 \\
1311\end{array}\right.$ & $\begin{array}{l}15 \\
13\end{array}$ & 1331 & 21 & $v \mathrm{C}-\mathrm{O}$ a. $(25)+v \mathrm{C}-\mathrm{C}$ a. $(23)$ \\
\hline$\delta \mathrm{CCH}$ a. & $\begin{array}{l}1217 \\
1211\end{array}$ & $\begin{array}{r}11 \\
7\end{array}$ & 1207 & 43 & $\delta \mathrm{CCH}$ a. $(83)$ & 1256 & 30 & 1222 & 4 & $\delta \mathrm{CCH}$ a. $(47)+\delta \mathrm{COH}$ a. $(40)$ \\
\hline$\delta \mathrm{COH}$ a. & $\left\{\begin{array}{l}1115 \\
1111\end{array}\right.$ & $\begin{array}{l}46 \\
10\end{array}$ & 1103 & 101 & $\delta \mathrm{COH}$ a. $(43)+v \mathrm{C}-\mathrm{O}$ a. $(28)$ & 1132 & 52 & 1128 & 63 & $\delta \mathrm{COH}$ a. $(22)+v \mathrm{C}-\mathrm{O}$ a. $(39)+\delta \mathrm{CCH}$ a. (29) \\
\hline$\gamma \mathrm{CH}$ & 982 & 8 & 988 & 6 & $\gamma \mathrm{CH}(99)$ & 988 & 9 & 995 & 4 & $\gamma \mathrm{CH}(99)$ \\
\hline$v \mathrm{C}-\mathrm{C}$ a. & 914 & 2 & 891 & 1 & $v \mathrm{C}-\mathrm{C}$ a. $(59)+v \mathrm{C}-\mathrm{O}$ a. $(23)$ & 902 & 1 & 883 & 1 & $v \mathrm{C}-\mathrm{C}$ a. $(66)+v \mathrm{C}-\mathrm{O}$ a. $(24)$ \\
\hline$\gamma \mathrm{C}=\mathrm{O}$ & 777 & 31 & 757 & 19 & $\gamma \mathrm{C}=\mathrm{O}(59)+\tau \mathrm{C}-\mathrm{O}(21)$ & 777 & 31 & 757 & 9 & $\gamma \mathrm{C}=\mathrm{O}(52)+\tau \mathrm{C}-\mathrm{O}(26)$ \\
\hline$\delta$ OCO a. & $\{600$ & 8 & 614 & 41 & $\delta$ OCO a. (68) & 560 & 6 & 577 & 17 & $\delta$ OCO a. $(65)$ \\
\hline & ¿ 599 & 30 & & & & 557 & 4 & 577 & 19 & $\tau \mathrm{C}-\mathrm{O}(80)$ \\
\hline$\tau \mathrm{C}-\mathrm{O}$ & 571 & 44 & 581 & 20 & $\tau \mathrm{C}-\mathrm{O}(87)$ & & & & & \\
\hline$\delta \mathrm{CC}=\mathrm{O}$ a. & 537 & 7 & 520 & 10 & $\begin{array}{l}\delta \mathrm{CC}=\mathrm{O} \text { a. }(55)+\delta \mathrm{CCC} \text { a. }(17)+ \\
\quad \delta \text { OCO a. }(16)\end{array}$ & 537 & 7 & 520 & 4 & $\delta \mathrm{CC}=\mathrm{O}$ a. $(53)+\delta$ OCO a. $(19)$ \\
\hline$\tau \mathrm{C}=\mathrm{C}$ & & & 141 & $<1$ & $\tau \mathrm{C}=\mathrm{C}(60)+\gamma \mathrm{CH}(30)$ & & & 137 & $<1$ & $\tau \mathrm{C}=\mathrm{C}(62)+\gamma \mathrm{CH}(31)$ \\
\hline$\delta$ CCC a. & & & 122 & $<1$ & $\delta \mathrm{CCC}$ a. $(77)+\delta \mathrm{CC}=\mathrm{O}$ a. $(21)$ & & & 133 & $<1$ & $\delta \mathrm{CCC}$ a. $(75)+\delta \mathrm{CC}=\mathrm{O}$ a. $(22)$ \\
\hline$\tau \mathrm{C}-\mathrm{C}$ & & & 52 & $<1$ & $\tau \mathrm{C}-\mathrm{C}(61)+\gamma \mathrm{CH}(22)$ & & & 48 & $<1$ & $\tau \mathrm{C}-\mathrm{C}(88)$ \\
\hline
\end{tabular}

${ }^{a}$ Frequencies in $\mathrm{cm}^{-1}$; intensities in $\mathrm{km} \mathrm{mol}^{-1}$; calculated frequencies were scaled by 0.9614 ; experimental intensity values presented correspond to the observed integrated intensities of each conformer normalized to 1000. Calculated intensities were scaled to experimental integrated normalized intensities (only bands which have an experimental counterpart and can be ascribable to a single conformer were used in the scaling procedure). ${ }^{b}$ Frequency values shown in bold represent bands assigned to more than one conformer. In these case, the experimental intensities shall be compared with the sum of the calculated intensities for the three observed conformers (see also Table 7 ). ${ }^{c}$ Only contributions higher than $15 \%$ are listed. ${ }^{d}$ Tentative assignment as combination band. $v$, stretching; $\delta$, bending; $\tau$, torsion; $\gamma$, rocking. Minor bands due to nonfundamental modes are also observed at 1808, 1794, 1348, 1320, 1301, 1190, 1106, 714, $712 \mathrm{~cm}^{-1}$.

inactive in conformers I and III belonging to the $C_{2 h}$ symmetry point group. The presence of conformers I and III in the matrix can also be clearly established as described below. It is interesting to note that a recent study on matrix isolated fumaryl chloride $[(E)-\mathrm{ClC}(=\mathrm{O})-\mathrm{C}=\mathrm{C}-(\mathrm{C}=\mathrm{O}) \mathrm{Cl}]$ succeeded to observe the three conformers of that molecule which are the analogous chlorine-substituted forms of the three most stable conformers of fumaric acid predicted by the calculations. ${ }^{29}$

In the $\mathrm{OH}$ stretching region, the band observed at the highest wavenumber $\left(3576 \mathrm{~cm}^{-1}\right)$ is assigned to conformers II and III, both having bands predicted to occur at $3620 \mathrm{~cm}^{-1}$ (Figure 4a). The two bands at lower wavenumbers $\left(\sim 3560 \mathrm{~cm}^{-1}\right)$ are assigned to the asymmetric $\mathrm{OH}$ stretching mode of conformer I and to the second $\mathrm{OH}$ stretching vibration of conformer II. Both vibrational modes are computed to lie at ca. $3610 \mathrm{~cm}^{-1}$. The broad band observed at $2948 \mathrm{~cm}^{-1}$ is attributed to the IR active stretching $\mathrm{C}-\mathrm{H}$ vibrations of the three conformers, predicted to lie in the $3115-3050 \mathrm{~cm}^{-1}$ region and being very weak.

As expected, taking into consideration the structural similarity of the three experimentally observed conformers of fumaric acid, their $\mathrm{C}=\mathrm{O}$ stretching modes appear as a "complex band" with six components separated by a few wavenumbers in the 1765 to $1750 \mathrm{~cm}^{-1}$ region. Although it was not possible to make a detailed assignment of this multiplet, the calculations indicate that its higher wavenumber components should be mainly due to the $\mathrm{C}=\mathrm{O}$ stretching mode of conformer II, which is predicted to occur at $1760 \mathrm{~cm}^{-1}$. The lower wavenumber components is suitable to the second vibration of this conformer (predicted at $1753 \mathrm{~cm}^{-1}$ ) as well as to the $\mathrm{C}=\mathrm{O}$ asymmetric stretching modes of conformers I and III (that are predicted at 1750 and 1754 $\mathrm{cm}^{-1}$, respectively).

The strongest support for the presence of three conformers of fumaric acid in the matrix can be found in the $\mathrm{C}-\mathrm{O}$ stretching region. The $\mathrm{C}-\mathrm{O}$ stretching in conformer $\mathrm{I}$ is predicted to occur at higher frequency $\left(1352 \mathrm{~cm}^{-1}\right)$ than for conformer III (1331 $\mathrm{cm}^{-1}$ ), while the two $\mathrm{C}-\mathrm{O}$ vibrations of conformer II are predicted to occupy intermediate wavenumbers (1345 and 1337 $\mathrm{cm}^{-1}$ ). The experimental spectrum exhibits the same pattern in this spectral region (Figure 4b; see also Tables 6 and 7). The band observed at $1369 \mathrm{~cm}^{-1}$ is ascribable to the $\mathrm{C}-\mathrm{O}$ stretching mode of conformer $\mathrm{I}$. The two $\mathrm{C}-\mathrm{O}$ stretching modes of conformer II give rise to the band at $1338 \mathrm{~cm}^{-1}$ and the doublet at 1364 and $1357 \mathrm{~cm}^{-1}$ (in the last case, the observed splitting results from matrix site effects). The lowest site-splitted band found at 1315 and $1311 \mathrm{~cm}^{-1}$ is due to conformer III.

The agreement between the predicted and experimental spectra is also quite clear in the in-plane $\mathrm{COH}$ bending region (Figure 4b), where the bands belonging to conformer II (predicted at 1154 and $1116 \mathrm{~cm}^{-1}$ ) are observed at 1158 and $1120 \mathrm{~cm}^{-1}$. The vibrational modes of conformers I and III (predicted at 1103 and $1128 \mathrm{~cm}^{-1}$, respectively) are observed at $1115-1111 \mathrm{~cm}^{-1}$ and $1132 \mathrm{~cm}^{-1}$.

NIR Irradiation and Annealing Experiments. Selective irradiation of the $\mathrm{OH}$ stretching vibrations have been demonstrated to be a very elegant way of promoting conformational isomerization processes in matrix isolated carboxylic compounds. ${ }^{24-26,30}$ The use of narrow band tunable irradiation in the NIR region, at a frequency of the first $\mathrm{OH}$ stretching overtone or a suitable combination was found to be particularly 
TABLE 7: Observed (Ar matrix) and Calculated B3LYP/6-31G(d,p) Vibrational Frequencies and IR Intensities ${ }^{a}$ of Conformer $^{-1}$ II of Fumaric Acid ( $C_{S}$ Symmetry)

\begin{tabular}{|c|c|c|c|c|c|}
\hline \multicolumn{6}{|c|}{ Conformer II } \\
\hline $\begin{array}{l}\text { aproximate } \\
\text { description }\end{array}$ & $v_{\text {exp }}$ & $I_{\exp }$ & $v_{\text {calc }}$ & $I_{\text {calc }}$ & $\mathrm{PED}^{b}$ \\
\hline$v \mathrm{O}-\mathrm{H}$ & 3576 & 22 & 3620 & 14 & $v \mathrm{O}-\mathrm{H}(100)$ \\
\hline$v \mathrm{O}-\mathrm{H}^{\prime}$ & 3560 & 27 & 3610 & 14 & $v \mathrm{O}-\mathrm{H}^{\prime}(100)$ \\
\hline$v \mathrm{CH}^{\prime}$ & 2948 & 27 & 3106 & $<1$ & $v \mathrm{CH}^{\prime}(89)$ \\
\hline$v \mathrm{CH}$ & & & 3101 & & $v \mathrm{CH}(88)$ \\
\hline \multirow[t]{4}{*}{$v \mathrm{C}=\mathrm{O}$} & 1765 & 16 & 1760 & 19 & $v \mathrm{C}=\mathrm{O}(47)+v \mathrm{C}=\mathrm{O}^{\prime}(26)$ \\
\hline & 1763 & 15 & 1753 & 67 & $v \mathrm{C}=\mathrm{O}^{\prime}(51)+v \mathrm{C}=\mathrm{O}(28)$ \\
\hline & 1759 & 29 & & & \\
\hline & 1756 & 84 & & & \\
\hline \multirow[t]{2}{*}{$v \mathrm{C}=\mathrm{O}^{\prime}$} & 1754 & 38 & & & \\
\hline & 1750 & 39 & & & \\
\hline$v \mathrm{C}=\mathrm{C}$ & 1650 & 18 & 1651 & 6 & $v \mathrm{C}=\mathrm{C}(66)$ \\
\hline \multirow[t]{2}{*}{$v \mathrm{C}-\mathrm{O}^{\prime}$} & $\{1364$ & 35 & 1345 & 33 & $v \mathrm{C}-\mathrm{O}^{\prime}(18)$ \\
\hline & 1357 & 8 & & & \\
\hline$v \mathrm{C}-\mathrm{O}$ & 1338 & 9 & 1337 & 10 & $v \mathrm{C}-\mathrm{O}(23)+\delta \mathrm{COH}(16)$ \\
\hline \multirow[t]{3}{*}{$\delta \mathrm{CCH}$} & 1274 & 3 & 1257 & $<1$ & $\delta \mathrm{CCH}(40)+\delta \mathrm{CCH}^{\prime}(24)$ \\
\hline & 1269 & $<1$ & & & \\
\hline & 1242 & 2 & & & $986+257^{d}$ \\
\hline$\delta \mathrm{CCH}^{\prime}$ & 1234 & 14 & 1213 & 12 & $\delta \mathrm{CCH}^{\prime}(26)+\delta \mathrm{CCH}(36)+\delta \mathrm{COH}^{\prime}(21)$ \\
\hline$\delta \mathrm{COH}$ & 1158 & 17 & 1154 & 48 & $\delta \mathrm{COH}(46)+v \mathrm{C}-\mathrm{O}(21)$ \\
\hline$\delta \mathrm{COH}^{\prime}$ & 1120 & 52 & 1116 & 50 & $\delta \mathrm{COH}^{\prime}(24)+v \mathrm{C}-\mathrm{O}^{\prime}(38)+\delta \mathrm{CCH}^{\prime}(16)$ \\
\hline$\gamma \mathrm{CH}$ & 986 & 11 & 994 & 6 & $\gamma \mathrm{CH}(65)+\gamma \mathrm{CH}^{\prime}(34)$ \\
\hline$v \mathrm{C}-\mathrm{C}^{\prime}$ & 938 & 4 & 917 & 3 & $v \mathrm{C}-\mathrm{C}^{\prime}(48)$ \\
\hline$\gamma \mathrm{CH}^{\prime}$ & 909 & 2 & 885 & $<1$ & $\gamma \mathrm{CH}^{\prime}(46)+\gamma \mathrm{CH}(36)$ \\
\hline$v \mathrm{C}-\mathrm{C}$ & 896 & 2 & 879 & $<1$ & $v \mathrm{C}-\mathrm{C}(41)+v \mathrm{C}-\mathrm{O}(36)$ \\
\hline$\gamma \mathrm{C}=\mathrm{O}^{\prime}$ & 777 & 31 & 757 & 14 & $\gamma \mathrm{C}=\mathrm{O}^{\prime}(30)+\gamma \mathrm{C}=\mathrm{O}(26)$ \\
\hline$\tau \mathrm{C}-\mathrm{O}^{\prime}$ & n. o. & & 667 & 2 & $\tau \mathrm{C}-\mathrm{O}^{\prime}(29)+\tau \mathrm{C}-\mathrm{O}(19)+\gamma \mathrm{C}=\mathrm{O}(19)$ \\
\hline$\delta \mathrm{OCO}^{\prime}$ & n. $o$. & & 640 & $<1$ & $\delta \mathrm{OCO}^{\prime}(35)+\delta \mathrm{OCO}(24)$ \\
\hline$\tau \mathrm{C}-\mathrm{O}$ & 589 & 50 & 592 & 34 & $\tau \mathrm{C}-\mathrm{O}(58)+\tau \mathrm{C}-\mathrm{O}^{\prime}(23)$ \\
\hline$\delta \mathrm{OCO}$ & 554 & 10 & 568 & 17 & $\delta \mathrm{OCO}(51)+\delta \mathrm{OCO}^{\prime}(31)$ \\
\hline$\gamma \mathrm{C}=\mathrm{O}$ & 546 & 3 & 544 & $<1$ & $\gamma \mathrm{C}=\mathrm{O}(29)+\gamma \mathrm{C}=\mathrm{O}^{\prime}(24)$ \\
\hline$\delta \mathrm{CC}=\mathrm{O}$ & & & 529 & 3 & $\delta \mathrm{CC}=\mathrm{O}(38)+\delta \mathrm{CC}=\mathrm{O}^{\prime}(28)$ \\
\hline$\delta \mathrm{CC}=\mathrm{O}^{\prime}$ & & & 371 & $<1$ & $\delta \mathrm{CC}=\mathrm{O}^{\prime}(30)+\delta \mathrm{CC}=\mathrm{O}(24)$ \\
\hline$\delta \mathrm{CCC}^{\prime}$ & & & 257 & $<1$ & $\delta \operatorname{CCC}^{\prime}(24)+\delta \operatorname{CCC}(20)$ \\
\hline$\tau \mathrm{C}=\mathrm{C}$ & & & 141 & $<1$ & $\tau \mathrm{C}=\mathrm{C}(65)+\gamma \mathrm{CH}(16)$ \\
\hline$\tau \mathrm{C}-\mathrm{C}^{\prime}$ & & & 138 & $<1$ & $\tau \mathrm{C}-\mathrm{C}^{\prime}(41)+\tau \mathrm{C}-\mathrm{C}(37)$ \\
\hline$\delta \mathrm{CCC}$ & & & 128 & $<1$ & $\delta \mathrm{CCC}(38)+\delta \mathrm{CCC}^{\prime}(38)$ \\
\hline$\tau \mathrm{C}-\mathrm{C}$ & & & 50 & $<1$ & $\tau \mathrm{C}-\mathrm{C}(50)+\tau \mathrm{C}-\mathrm{C}^{\prime}(27)$ \\
\hline
\end{tabular}

${ }^{a}$ Frequencies in $\mathrm{cm}^{-1}$; intensities in $\mathrm{km} \mathrm{mol}^{-1}$; calculated frequencies were scaled by 0.9614 ; experimental intensity values presented correspond to the observed integrated intensities of each conformer normalized to 1000. Calculated intensities were scaled to experimental integrated normalized intensities (only bands which have an experimental counterpart and can be ascribable to a single conformer were used in the scaling procedure). ${ }^{b}$ Frequency values shown in bold represent bands assigned to more than one conformer. In these case, the experimental intensities shall be compared with the sum of the calculated intensities for the three observed conformers (see also Table 6). ${ }^{c}$ Only contributions higher than $15 \%$ are listed. $v$, stretching; $\delta$, bending; $\tau$, torsion; $\gamma$, rocking, ', refers to $\mathrm{O}_{8}=\mathrm{C}_{4}\left(-\mathrm{O}_{9}-\mathrm{H}_{10}\right)-\mathrm{C}_{3}\left(-\mathrm{H}_{12}\right)$ fragment.Minor bands due to nonfundamental modes are also observed at 1808, 1794, 1348, 1320,1301, 1190, 1106, 714, $712 \mathrm{~cm}^{-1}$.

efficient in initiating the conformational isomerization of matrixisolated oxalic and malonic acids. ${ }^{22,25}$ In the present study NIR irradiation of matrix-isolated maleic and fumaric acids, in the $7020-6830 \mathrm{~cm}^{-1}$ region, was undertaken.

For maleic acid, the irradiation experiments unequivocally confirmed the computational results with respect to the most stable conformers. Irradiation of the matrix at $6901 \mathrm{~cm}^{-1}$ led to an increase of the bands assigned to conformer I, while bands due to conformer II decreased (see Figure 3), which indicates that the NIR-irradiation is effective in promoting interconversion from conformer II to the most stable conformer I.

In the case of fumaric acid, irradiation all over the region covered $\left(7018-6873 \mathrm{~cm}^{-1}\right)$ was found to be inefficient to initiate any conformational isomerization process. Note that the first overtone of the $\mathrm{OH}$ stretching mode in fumaric acid is expected to lie in the $6996-6940 \mathrm{~cm}^{-1}$ region, taking into account the results of previous studies undertaken on malonic acid. ${ }^{22}$ In fact, it has been shown that in this kind of molecules anharmonicity associated with the $\mathrm{OH}$ stretching vibration may account for a red shift of ca. $160 \mathrm{~cm}^{-1}$ in the position of its first overtone when compared with twice the fundamental frequency.

The observed inefficiency of irradiation at the first overtone of the $\mathrm{OH}$ stretching mode in fumaric acid to promote interconversion between the low-energy observed conformers may have the same origin as the previously reported failure to promote by this way isomerization reactions implying internal rotation around the $\mathrm{C}-\mathrm{C}$ central bond in oxalic acid. ${ }^{25}$ This was explained on the basis of the extensive matrix reorganization required by internal rotation about the $\mathrm{C}-\mathrm{C}$ bond, which involves a relatively large motion of the molecular fragments. This rearrangement leads to considerably higher effective energy barriers when compared with the molecule in the vacuum. ${ }^{25}$ Further support to this interpretation can also be taken from recent studies on matrix isolated oxalyl fluoride and nitrous acid, ${ }^{31,32}$ which clearly demonstrated that isomerization processes induced by narrowband IR radiation can be selective with respect to the matrix sites, and no significant matrix rearrangements occur. Note that in maleic acid the observed intercon- 

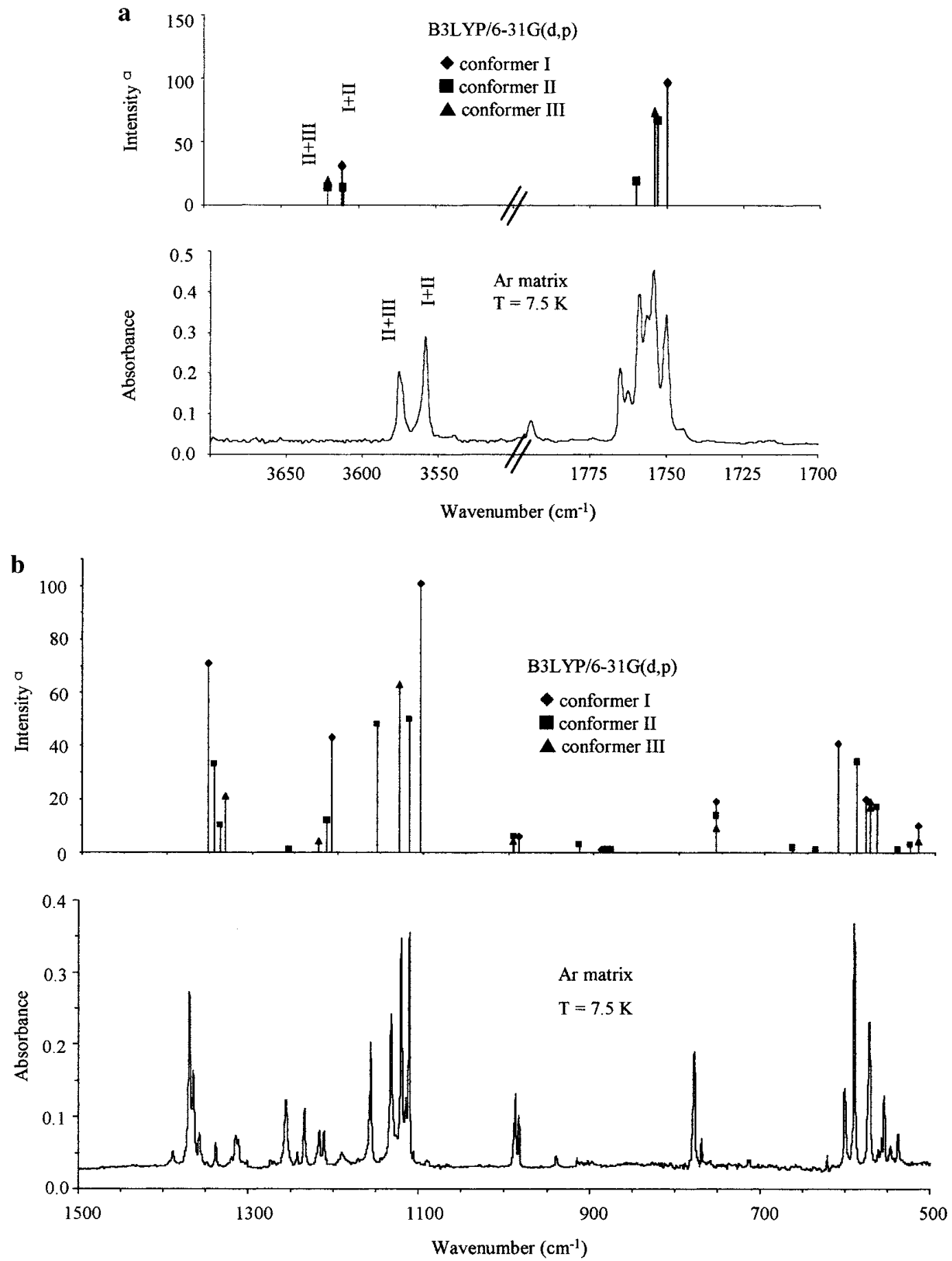

Figure 4. IR spectrum of fumaric acid in an argon matrix $(T=7.5 \mathrm{~K})$ and predicted (B3LYP) spectra for the three lowest energy conformers: (a) $3700-1700 \mathrm{~cm}^{-1}$ region and (b) $1500-500 \mathrm{~cm}^{-1}$ region. ${ }^{a}$ Calculated intensities as shown in Tables 6 and 7 where the normalization procedure is explained in detail.

version processes involve only small adjustments in the positions of the heavy atoms and so, contrary to the rotamerization processes in fumaric acid, these processes do not require any significant matrix rearrangements.

The lack of NIR induced isomerization processes in fumaric acid might also be attributed to the absence of any suitable mechanism for energy transfer between the excited vibrational mode and the reaction coordinates ( $\nu \mathrm{OH}$ overtone and the $\tau$ $\mathrm{C}-\mathrm{C}$, respectively) or weak absorption by the overtone. Nevertheless, the fact that broadband IR irradiation was also unable to promote any observable isomerization process in fumaric acid seems to indicate that, as discussed above, relatively high energy barriers for conformational isomerization in fumaric acid (associated in some extent with matrix relaxation) shall, at least in part, explain the different behavior of the two studied compounds.

UV irradiation $(\lambda=266 \mathrm{~nm})$ of fumaric acid in argon matrixes was also performed. As shown in Figure 5, UV excitation efficiently promotes isomerization of fumaric acid to maleic acid. On the other hand, no detectable conformational interconversion between the observed conformers of fumaric acid could be observed. A similar photochemically induced tautomerization reaction was also previously observed for matrix isolated fumaryl chloride $(\lambda=340 \mathrm{~nm}) .{ }^{29}$ In this case, maleoyl chloride was produced, and, as for fumaric acid, no conforma- 

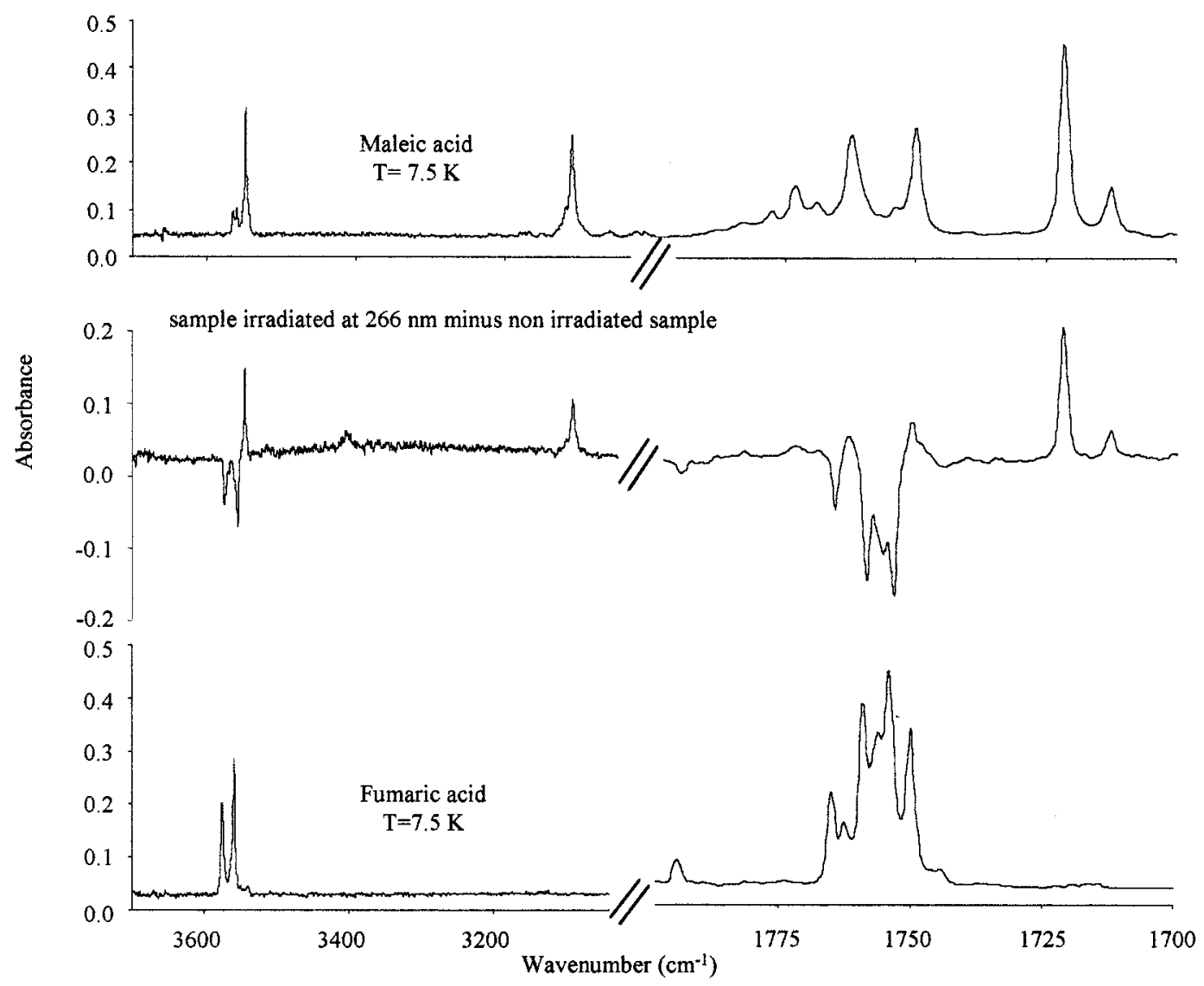

Figure 5. Matrix isolation IR spectra of maleic (top) and fumaric (bottom) acids and difference spectra obtained by subtracting to the spectrum of a freshly prepared matrix of fumaric acid from the spectrum of the same sample after UV irradiation at $266 \mathrm{~nm}$ (middle).

tional interconversion between the conformers initially present in the matrix could be observed.

We have also studied the thermally induced conformational isomerization for maleic and fumaric acids by annealing freshly prepared matrixes up till $35 \mathrm{~K}$. In the case of maleic acid, a noticeable redistribution of intensities starts at $22 \mathrm{~K}$, clearly evidencing an increase of the relative population of the conformational ground state (Figure 3). Taking into consideration the relationship first derived by Barnes, ${ }^{33}$ these results point to an energy barrier for conversion of conformer II into conformer I of ca. $6 \mathrm{~kJ} \mathrm{~mol}^{-1}$. For fumaric acid, no changes in the spectrum attributed to rotamerization processes were observed within the experimentally accessible temperature range. This result is an additional support for the interpretation based on energy considerations, above made, to explain the absence of any photoisomerization reaction in fumaric acid. In addition, it also indicates that the energy barriers for conformer interconversion in this molecule are higher than $12 \mathrm{~kJ} \mathrm{~mol}^{-1}$, the largest accessible barrier at the maximum temperature attained. ${ }^{33}$ The MP2 calculated energy barriers associated with the studied conformational isomerization processes for fumaric acid in a vacuum amount to ca. $20 \mathrm{~kJ} \mathrm{~mol}^{-1}$.

Experimental vs Calculated Populations of the Observed Conformers. Relative energies of the observed conformers can be calculated from their relative populations at a given temperature assuming a Boltzmann distribution. The relative populations of the observed conformers can be estimated from the experimental IR band intensities recorded immediately after deposition taking into account the calculated intensities and assuming that no conformational isomerization takes place during the deposition process. For maleic acid the $\mathrm{O}-\mathrm{H}$ and $\mathrm{C}=\mathrm{O}$ stretching regions were used in these estimations due to the intense signals observed, as well as because these regions are not congested and can unequivocally be used to assign the different conformers. For fumaric acid, the $\mathrm{C}-\mathrm{O}$ stretching, inplane $\mathrm{COH}$ bending and $\mathrm{C}=\mathrm{O}$ rocking spectral regions were used by the same reasons.

The energy difference between the two observed conformers of maleic acid obtained by this method is $1.7 \mathrm{~kJ} \mathrm{~mol}^{-1}$, which is in excellent agreement with the MP2 predictions $(1.3 \mathrm{~kJ}$ $\mathrm{mol}^{-1}$ ). For fumaric acid, a good qualitative agreement between the $\mathrm{ab}$ initio and the experimentally determined relative energies of the conformers was also obtained, although the theoretical predictions at all levels used systematically give higher values (measured, $E_{\mathrm{II}}-E_{\mathrm{I}}=0.7 \mathrm{~kJ} \mathrm{~mol}^{-1}$ and $E_{\mathrm{III}}-E_{\mathrm{I}}=0.9 \mathrm{~kJ}$ $\mathrm{mol}^{-1}$; MP2, $E_{\mathrm{II}}-E_{\mathrm{I}}=2.3 \mathrm{~kJ} \mathrm{~mol}^{-1}$ and $E_{\mathrm{III}}-E_{\mathrm{I}}=4.6 \mathrm{~kJ}$ $\left.\mathrm{mol}^{-1}\right)$.

\section{Conclusion}

The ab initio calculations carried out at the MP2 and B3LYP levels were used to characterize the most stable conformers of maleic and fumaric acids and the computational results were used to aid the interpretation of the infrared spectra of these compounds isolated in an argon matrix. The lowest energy conformer of maleic acid was found to exhibit a nearly planar structure with an $\mathrm{OH} \cdots \mathrm{O}=\mathrm{C}$ intramolecular hydrogen bond closing a seven member $\mathrm{O}=\mathrm{C}-\mathrm{C}(\mathrm{H})=\mathrm{C}(\mathrm{H})-\mathrm{C}-\mathrm{O}-\mathrm{H}$ ring. The second conformer of maleic acid adopts a cis arrangement around the $\mathrm{C}-\mathrm{O}$ bonds and exhibits two carbonyl bonds in a nearly orthogonal arrangement with one carboxylic group in the plane containing the carbon atoms. For fumaric acid, three low-energy conformers exhibiting a cis arrangement around the $\mathrm{C}-\mathrm{O}$ bond where found computationally and experimentally. 
The conformers differ by the relative conformation of their $\mathrm{O}=\mathrm{C}-\mathrm{C}=\mathrm{C}$ axes and were predicted to be almost equal energetically.

The use of narrow band tunable NIR irradiation at the first $\mathrm{OH}$ stretching overtone together with the annealing of the matrix up to $35 \mathrm{~K}$ allowed us to directly observe conformational isomerization between the low-energy conformers of matrixisolated maleic acid. On the other hand, no conformational interconversions were observed for fumaric acid by vibrational excitation (both in the NIR and mid-IR regions) or annealing. This points to relatively high energy barriers to internal rotation in matrix-isolated fumaric acid. A lower limit of ca. $12 \mathrm{k} \mathrm{J}$ $\mathrm{mol}^{-1}$, resulting from the annealing studies, can be estimated for these barriers in fumaric acid, this value being nearly twice the energy barrier associated with the observed rotamerization processes in maleic acid.

Acknowledgment. The authors acknowledge financial support from Academy of Finland. R.F. also thanks financial support from Fundação para a Ciência e a Tecnologia, Lisbon, Portugal (research project PRAXIS/P/QUI/10137/1998).

\section{References and Notes}

(1) Muller. Corros. Sci. 1995, 37, 877.

(2) Solich, M.; Krol, W.; Skirmuntt, K. Pol. J. Chem. 1993, 67, 433.

(3) Kawaguchi, S.; Kitano, T.; Ito, K. Macromolecules 1992, 25, 1294. 2477.

(4) Wang, F. C.; Green, J. G.; Gerhart, B. B. Anal. Chem. 1996, 68,

(5) Eiem-Ong, S.; Spohn, M.; Kurtzman, N. A.; Sabatini, S. Kidney Int. 1995, 48, 1542 .

(6) Castrano, E. Clin. Sci. 1997, 92, 247.

(7) a) Tsuchiya, K.; Uchida, T.; Kobayashi, M.; Maeda, H.; Konno, T.; Yamanaka, H. Urology 2000, 55 (4), 495. (b) Abe, S.; Okubo, Y.; Ejiri,

Y.; Kume, K; Otsuki, M. J. Gastroenterol. 2000, 35 (1), 28.

(8) Haan, P.; Blomberg-van der Flier, B. M. E, Groot, J.; Nieboer, C; Bruynzeel, D. P. Dermatology 1994, 188, 126.

(9) Akao, M.; Kuroda, K. Chem. Pharm. Bull. 1990, 38, 2012.

(10) Shahat, M. Acta Crystallogr. 1952, 5, 763.

(11) Brown, C. J. Acta Crystallogr. 1966, 21, 1.

(12) Bednowitz, A. L.; Post, B. Acta Crystallogr. 1966, 21, 566

(13) Kulbida, A.; Ramos, M. N.; Räsänen, M.; Nieminen, J.; Schrems,

O.; Fausto, R. J. Chem., Soc. Faraday Trans. 1995, 91, 1571.

(14) Fausto, R.; Kulbida, A.; Schrems, O. J. Chem. Soc., Faraday Trans. 1995, 91, 3755 .

(15) Fausto, R.; Maçôas, E. M. S. J. Mol. Struct. 1999, 480-481, 83.

(16) Hehre, J. W.; Ditchfield, R.; Pople, J. A. J. Chem. Phys., 1972, $56,2257$.
(17) a) Møller, C.; Plesset, M. S.; Phys. Rev. 1934, 46, 618. (b) Binkley, J. S.; Pople, J. A. Int. J. Quantum Chem. 1975, 9, 229.

(18) a) Becke, A. D. J. Chem. Phys. 1993, 98, 5648. (b) Lee, C.; Yang, W.; Parr, R. G. Phys. Rev. 1988, B37, 785; Miehlich, B.; Savin, A.; Stoll, H.; Preuss, H.; Chem. Phys. Lett. 1989, 157, 200.

(19) a) Frisch, M. J.; Trucks, G. W.; Schlegel, H. B.; Gill, P. M. W. Johson, B. G.; Wong, M. W.; Foresman, J. B.; Robb, M. A.; Head-Gordon, M.; Replogle, E. S.; Gomperts, R.; Andres, J. L.; Raghavachari, K.; Binkley, J. S.; Gonzalez, C.; Martin R. L.; Fox, D. J.; Defrees, D. J.; Baker, J.; Stewart, J. J. P.; Pople, J. A. GAUSSIAN92/DFT (rev. G.2), Gaussian Inc., Pittsburgh, PA, 1993. (b) Frisch, M. J.; Trucks, G. W.; Schlegel, H. B. Gill, P. M. W.; Johson, B. G.; Robb, M. A.; Cheeseman, J. R.; Keith, T.; Petersson, G. A.; Montgomery, J. A.; Raghavachari, K.; Al-Laham, M. A.; Zakrzewski, V. G.; Ortiz, J. V.; Foresman, J. B.; Cioslowski, J.; Stefanov, B. B.; Nanayakkara, A.; Challacombe, M.; Peng, C. Y.; Ayala, P. Y.; Chen, W.; Wong, M. W.; Andres, J. L.; Reploge, E. S.; Gomperts, R.; Martin, R. L.; Fox, D. J.; Binkley, J. S.; Defrees, D. J.; Baker, J.; Stewart, J. J. P.; Head-Gordon, M.; Gonzalez, C.; Pople, J. A. GAUSSIAN 94; Gaussian Inc., Pittsburgh, PA, 1995; Revision B.1.

(20) a) Binkley, J. S.; Pople, J. A.; Hehre, W. J. J. Am. Chem. Soc. 1980, 102, 939. (b) Gordon, M. S.; Binkley, J. S.; Pople, J. A.; Pietro, W. J.; Hehre, W. J. J. Am. Chem. Soc. 1982, 104, 2797.

(21) Hariharan, P. C.; Pople, J. A. Theor. Chim. Acta (Berl.) 1973, 28, 213.

(22) Maçôas, E. M. S.; Fausto, R.; Lundell, J.; Pettersson, M.; Khriachtchev, L.; Räsänen, M, J. Phys. Chem. A 2000, 104, 11725.

(23) Fausto, R. TRANSFORMER (version 2.0); Chemistry Department, University of Coimbra, Portugal, 1997.

(24) Faria, M. D. G.; Fausto R. BUILD-G (version 2.0) and VIBRAT (version 2.0); Chemistry Department, University of Coimbra, Portugal, 1997 (these programs incorporate several routines from programs GMAT and FPERT, Fuher, H.; Kartha, V. B.; Krueger, P. J.; Mantsch, H. H. Natl. Res. Council Can. Bull. 1976, 15, 1.)

(25) Maçôas, E. M. S.; Fausto, R.; Pettersson, M.; Khriachtchev, L.; Räsänen, M. J. Phys. Chem A 2000, 104, 6956.

(26) Fausto, R. Low-Temperature Molecular Spectroscopy; Fausto, R., Ed.; NATO-ASI Series C483, Kluwer Academic: Amsterdam (The Netherlands), 1996; 125.

(27) Fausto, R. Theochemistry 1994, 315, 123.

(28) Batista de Carvalho, L. A. E.; Teixeira-Dias, J. J. C.; Fausto, R. Theochem., 1990, 208, 109; Teixeira-Dias, J. J. C.; Batista de Carvalho, L. A. E.; Fausto, R. J. Comput. Chem. 1991, 12, 1047.

(29) Piétri, N.; Jurca, B.; Monnier, M.; Hillebrand, M.; Aycard, J. P. Spectrochim. Acta 1999, 56A, 157 5303.

(31) Sander, S.; Willner, H.; Khriachtchev, L.; Pettersson, M.; Räsänen, M.; Varetti, L. J. Mol. Spectrosc. 2000, 203, 145.

(32) Khriachtchev, L.; Lundell, J.; Isoniemi, E.; Räsänen, M. J. Chem. Phys. 2000, 113, 4265.

(33) Barnes, A. J. J. Mol. Struct. 1984, 113, 161. 